\title{
Genetic interactions derived from high-throughput phenotyping of 6589 yeast cell cycle mutants
}

\author{
Jenna E. Gallegos ${ }^{1,7}$, Neil R. Adames ${ }^{1,6,7}$, Mark F. Rogers ${ }^{2}$, Pavel Kraikivski ${ }^{3}$, Aubrey Ibele ${ }^{1}$, Kevin Nurzynski-Loth ${ }^{1}$, Eric Kudlow ${ }^{1}$,
} T. M. Murali ${ }^{4}$, John J. Tyson (iD) ${ }^{5}$ and Jean Peccoud ${ }^{1,2 \bowtie}$

Over the last 30 years, computational biologists have developed increasingly realistic mathematical models of the regulatory networks controlling the division of eukaryotic cells. These models capture data resulting from two complementary experimental approaches: low-throughput experiments aimed at extensively characterizing the functions of small numbers of genes, and largescale genetic interaction screens that provide a systems-level perspective on the cell division process. The former is insufficient to capture the interconnectivity of the genetic control network, while the latter is fraught with irreproducibility issues. Here, we describe a hybrid approach in which the 630 genetic interactions between 36 cell-cycle genes are quantitatively estimated by highthroughput phenotyping with an unprecedented number of biological replicates. Using this approach, we identify a subset of highconfidence genetic interactions, which we use to refine a previously published mathematical model of the cell cycle. We also present a quantitative dataset of the growth rate of these mutants under six different media conditions in order to inform future cell cycle models.

npj Systems Biology and Applications (2020)6:11; https://doi.org/10.1038/s41540-020-0134-z

\section{INTRODUCTION}

Eukaryotic cells grow and divide using a highly conserved and integrated network of positive and negative controls that ensure genomic integrity and maintain cell size within reasonable bounds. Proper control of the cell division cycle is essential for competitive fitness, embryonic development and maturation, and tissue homeostasis. Failure in these control mechanisms may result in cell death, developmental defects, tissue dysplasia, or cancers. One of the foremost model organisms for unraveling the molecular mechanisms of cell cycle control is the budding yeast Saccharomyces cerevisiae. Several hundred yeast mutants, generated in dozens of research laboratories over the past 40 years, have led to the discovery and characterization of many genes and proteins that regulate progression through the cell cycle ${ }^{1}$. Because of the intense labor involved in these experiments, individual laboratories have tended to focus on small numbers of genes and proteins involved in subsections of the extensive network of gene/ protein interactions that control cell cycle events. This reductionist approach was necessary in the early stages of identifying and characterizing the molecular regulatory system, but it carries with it the danger of missing higher levels of network organization and their phenotypic consequences ${ }^{2-4}$.

In contrast to a detailed, reductionist experimental approach, which builds a regulatory network from the bottom up, a systemslevel approach seeks to provide a more global and less biased view of regulatory networks. Systems biologists can uncover key regulatory interactions and network architectures that bottom-up practitioners may have missed ${ }^{5,6}$. Unfortunately, the top-down, pan-genome approach, while good for generating hypotheses, is usually poor for testing hypotheses because the experiments are mostly correlative, and the data is often plagued by problems of accuracy and reproducibility. Combining a variety of 'omics' studies may help to overcome these challenges, but it is often difficult to integrate disparate data sets into a single network model $^{7-10}$. Ideally, one should combine top-down and bottom-up data, but huge discrepancies of scale between these two data types present barriers to integrating and understanding the hypotheses derived from each approach ${ }^{11-17}$.

To mitigate these problems, many researchers, including ourselves, have developed detailed mathematical models that integrate top-down and bottom-up approaches in order to describe the molecular mechanisms that underlie cell cycle regulation in budding yeast ${ }^{4,17-22}$. The governing equations of the model are simulated on a computer, and the model (the 'wiring diagram' of molecular interactions) is adjusted until it generates dynamic behaviors that reflect the documented molecular changes and general network behaviors observed in cells (e.g., cell viability, timing of cell cycle events, cell size at birth, and response to DNA damage or chromosome misalignment at mitosis) ${ }^{23-26}$. Often, the documented data is missing detailed molecular information, such as protein concentrations and rate constants of crucial reactions, but fitting the model (i.e., finetuning the parameter values) to extensive sets of phenotypic data usually introduces strong constraints on these unknown parameters ${ }^{19,27}$. In this way, mathematical models can refine our understanding of the molecular mechanisms underlying cell cycle progression and test if the proposed network architecture and kinetic rate-constant estimates are consistent with both bottomup and top-down observations.

One of the major problems when developing large mathematical models of the cell cycle has been the lack of consistent data sets. It has been challenging to compare data collected on cell cycle mutants that often have different genetic backgrounds, whose phenotypes are usually descriptive rather than quantitative, and whose phenotypes are assessed under inconsistent conditions. These problems leave the modeler with the difficult

\footnotetext{
${ }^{1}$ Colorado State University, Chemical and Biological Engineering, Fort Collins, CO, USA. ${ }^{2}$ GenoFAB, Inc., Fort Collins, CO, USA. ${ }^{3}$ Virginia Tech, Academy of Integrated Sciences, Blacksburg, VA, USA. ${ }^{4}$ Virginia Tech, Computer Science, Blacksburg, VA, USA. ${ }^{5}$ Virginia Tech, Biological Sciences, Blacksburg, VA, USA. ${ }^{6}$ Present address: New Culture, Inc., San Francisco, CA, USA. ${ }^{7}$ These authors contributed equally: Jenna E. Gallegos, Neil R. Adames. ${ }^{\bowtie}$ email: jean.peccoud@colostate.edu
} 
task of curating, interpreting and consolidating inconsistent, and sometimes unreliable experimental results.

A particularly pernicious example of this problem is the use of the 'synthetic lethal' (SL) phenotype of double-mutant yeast cells in the development and calibration of mathematical models of the budding yeast cell cycle. Synthetic lethality arises when viable yeast strains carrying deletions of two different genes are crossed to produce inviable, double-mutant progeny (i.e., gene1 $\Delta$ and gene2 $\Delta$ mutant strains are viable separately, but the doublemutant gene1 $\Delta$ gene2 $\Delta$ strain is inviable). Because they impose strong constraints on the control system, SL gene combinations are exceptionally useful in deducing the network wiring diagram and estimating the rate constants in the mathematical model. On the other hand, if the incomplete or inaccurate identification of SL combinations of genes can wreak havoc on a model by forcing the modeler to make adjustments that are unwarranted. Problems arise because the experimental identification of SL gene combinations is plagued by false-positives and false-negatives and by the fact that some synthetic-lethal interactions are dependent on the genetic background of the parental strain. Hence, for the purpose of modeling cell cycle control in budding yeast, it is crucial to have a reliable, well documented, independently confirmed set of SL gene combinations observed in a uniform genetic background.

We have addressed this problem by reconsidering the identification of SL gene combinations of 'cell-cycle control' genes in budding yeast through a disciplined construction of replicate double-mutant strains based on a synthetic gene array (SGA) technology ${ }^{28}$ pioneered by Tong and Boone ${ }^{29}$ and the E-MAP 28 workflow described by Schuldiner ${ }^{30}$.

We focused on a set of only 36 cell cycle genes, most of which are functionally well-characterized (Fig. 1). This list comprises all the nonessential genes included in a recent mathematical model of the yeast cell cycle (herein referred to as the 'Kraikivski' model $)^{19}$, as well as genes whose protein products have redundant functions or interact with the proteins represented in the model. In order to estimate the reproducibility of our data, we performed four different crosses to produce each of the 630 double mutants and we tested both mating types. We managed to produce and characterize a library of 6589 genetically distinct yeast strains. The unprecedented number of biological replicates included in this library and the variability of the phenotypic data it produced are raising new modeling challenges.

We first analyze the variability of SL screen results in our library and compare it with previously published SL interactions listed on The Saccharomyces Genome Database $(S G D)^{31}$, and then we validate our conclusions by tetrad analysis (TA). We generate lists of 'high confidence' and 'low confidence' SL interactions. Next, we compare these high-confidence SL interactions with the predictions of our most recent and extensive mathematical model of budding-yeast cell-cycle controls ${ }^{19}$. We find that, in its present state, the model's predictions of SL interactions are not very accurate because the predictions were based on parameter values estimated from a collection of SL gene combinations that misidentified some crucial genetic interactions. From our new collection of high-confidence and low-confidence SL gene combinations we reparametrize the model to get much better agreement with the data. We expect this newly parametrized version of the model will give more reliable predictions about the phenotypes of other types of budding yeast mutants as well.

Furthermore, we characterized the growth rates of our mutants under six different media conditions expected to differentially influence cell cycle progression, providing quantitative fitness data that can be used in the future development of more refined and stochastic models of the cell cycle.

\section{RESULTS}

Identification of SL interactions

To assess all possible combinations of 36 cell cycle knockouts across multiple biological replicates, we generated 8 sets of independent parent lines to be used in 4 crosses. To avoid suppressor mutations - a feature of the commercial yeast haploid gene deletion collections-we generated 110 parent strains by tetrad dissection of commercial heterozygous diploid gene deletion strains (either before or after switching the kanMX marker to natMX), and we generated four parent strains by de novo gene deletion in BY4741 or BY4742. Neither the commercial SSA1/ssa1A strain nor any diploids produced by crosses with any de novo ssa1 $\Delta$ mutant parent were able to sporulate, indicating that two copies of this HSP70 chaperone gene is essential for meiosis. Interestingly this was not the case for the Ssa1 cochaperone, Ydj1. We also generated SGA haploid selection marker strains by mating and tetrad dissection of the aforementioned strains with the SGA strain developed by the Boone lab ${ }^{29}$ or by de novo gene deletion in that strain (55 and 70 parent strains, respectively). Each set of parent strains carried at least two differently marked deletions in all or most of the 36 genes for each of two different markers. According to the workflow described in Fig. 2, single mutant parent strains with opposite markers were crossed, and both MATa and MATa using $\mathrm{SGA}^{29}$ haploid selection markers. Overall we performed four series of $36^{*} 36$ crosses and collected both mating types for each crosses. Out of the 10,368 cell lines that experimental design could generate, we obtained 6589 mutants corresponding to $36^{*} 35 / 2=630$ double-mutant combinations.

Examining these 6589 mutants, we first flagged potential SL interactions by scoring each cross as 'growth' or 'non-growth', i.e., each double-mutant haploid colony as 'present' or 'absent' on double mutant haploid selection plates (Fig. 3).

No combination of genes produced the same results in every cross. In fact, the results among biological replicates varied considerably (Fig. 3, Table S4). Hence, we set a threshold for defining likely SL interactions. If evidence for synthetic lethality was observed four or more times irrespective of which parent strain the deletions were derived from, we flagged the combination as 'likely SL' in Fig. 4. The complete results for all progeny are compiled in Fig. 5.

A threshold of four was selected, because it ensures that the interaction was seen in at least two of the independent sets of crosses performed. This threshold also provided for the highest level of agreement between our screen and previously published results (discussed in the following section). For our set of 630 combinations, we observed 29 that exhibited synthetic lethality in at least four biological replicates.

Comparing the results of our screen with previously reported SL interactions

In Fig. 4, we compare our results to the $36 \mathrm{SL}$ gene combinations documented on SGD for the 36 genes in our data set (excluding several curation errors which are listed in the supplement) and to the predictions of Kraikivski's published model ${ }^{19}$. There are 58 lines in Fig. 4, referring to 58 (out of 630 possible) gene combinations for which one or more of the following statements is/are true:

1. the combination is documented as SL on SGD,

2. the combination is observed in our screen as likely $\mathrm{SL}$, and

3. the combination has been predicted to be SL by Kraikivski's model.

A Venn diagram indicating the overlap of these 58 gene combinations is provided in Fig. $5 \mathrm{~b}$.

Of the 36 gene combinations documented as SL on SGD, 16 were observed as likely SL in our screen (Fig. 5b). Meanwhile, 13 of 


\begin{tabular}{|c|c|c|c|c|c|}
\hline Gene & Function & Gene & Function & $\mathrm{Ge}$ & e Function \\
\hline 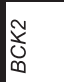 & Pos regulator of START. & $\sum_{U}^{N}$ & $\begin{array}{l}\text { Pos regulator of G1-S. G1 cyclin } \\
\text { activator of Cdc28. Paralog of CLN1. }\end{array}$ & $\frac{\bar{s}}{\omega}$ & $\begin{array}{l}\text { Neg regulator of G1-S. } \\
\text { Stoichiometric inhibitor of CDK } \\
\text { (CKI) Cdc28-Clb complexes. }\end{array}$ \\
\hline$\frac{\substack{\mathbf{x} \\
\infty}}{\infty}$ & $\begin{array}{l}\text { Neg regulator of M-G1. Spindle } \\
\text { Position Checkpoint (SPOC). } \\
\text { Subunit of Tem1 GTPase-activating } \\
\text { protein with Bub2. }\end{array}$ & $\sum_{0}^{m}$ & $\begin{array}{l}\text { Pos regulator of START. Promotes } \\
\text { transcription of CLN1 and CLN2. G1 } \\
\text { cyclin activator of Cdc28. CLN2 } \\
\text { paralog. }\end{array}$ & ङ & $\begin{array}{l}\text { Pos regulator of START. Cytosolic } \\
\text { HSP70 protein. Forms co- } \\
\text { chaperone complex with Ydj1. } \\
\text { Involved in reversing sequestration } \\
\text { of CLN3 mRNAs by Whi3, and } \\
\text { stress-response. }\end{array}$ \\
\hline$\underset{\infty}{\infty}$ & $\begin{array}{l}\text { Neg regulator of Metaphase- } \\
\text { Anaphase. Spindle Assembly } \\
\text { Checkpoint (SAC). Forms complex } \\
\text { with Bub3 to catalyze formation of } \\
\text { Mad-Cdc20 Mitotic Checkpoint } \\
\text { Complex (MCC). }\end{array}$ & $\frac{1}{\frac{1}{4}}$ & $\begin{array}{l}\text { Pos regulator of G2-M among other } \\
\text { chromatin functions. Transcription factor } \\
\text { that is partially redundant with FKH2, } \\
\text { but they often have opposite effects. }\end{array}$ & के & $\begin{array}{l}\text { Neg regulator of G2-M. } \\
\text { Morphogenesis Checkpoint. Protein } \\
\text { kinase phosphorylates and inhibits } \\
\text { Cdc28-Clb. Homolog of S. pombe } \\
\text { Wee1. }\end{array}$ \\
\hline 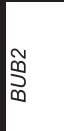 & $\begin{array}{l}\text { Neg regulator of M-G1. Spindle } \\
\text { Position Checkpoint (SPC). Subunit } \\
\text { of Tem1 GTPase-activating protein } \\
\text { with Bfa1. }\end{array}$ & $\frac{\sqrt{2}}{\frac{1}{2}}$ & $\begin{array}{l}\text { Pos regulator of G2-M among other } \\
\text { chromatin functions. Transcription factor } \\
\text { that is partially redundant with } F K H 1 \text {, } \\
\text { but they often have opposite effects. }\end{array}$ & क & $\begin{array}{l}\text { Pos regulator of START. } \\
\text { Transcription factor for late G1 } \\
\text { genes. Forms complex with Swi6 to } \\
\text { form SBF, which is partially } \\
\text { redundant with MBF. }\end{array}$ \\
\hline 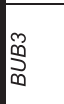 & $\begin{array}{l}\text { Neg regulator of Metaphase- } \\
\text { Anaphase. SAC. Forms complex } \\
\text { with Bub1 to catalyze formation of } \\
\text { Mad-Cdc20 MCC. }\end{array}$ & ๓ิ & $\begin{array}{l}\text { Neg regulator of M-G1. SPC. Interacts } \\
\text { with Bfa1 and epistatic to Bfa1-Bub2. }\end{array}$ & $\sum_{\omega}^{\infty}$ & $\begin{array}{l}\text { Pos regulator of } \mathrm{M}-\mathrm{G} 1 \text {. } \\
\text { Transcription factor for late } \mathrm{M} \text { and } \\
\mathrm{G} 1 \text { genes. }\end{array}$ \\
\hline 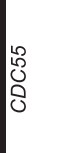 & $\begin{array}{l}\text { Pos regulator of G2-M and SAC } \\
\text { signaling. Inhibits M-G1. Regulatory } \\
\text { subunit of protein phosphatase 2A } \\
\text { (PP2A). Homolog of mammalian } \\
\text { B55. }\end{array}$ & 岕 & $\begin{array}{l}\text { Positive regulator of M-G1. Unknown } \\
\text { function, but epistatic to mitotic exit } \\
\text { network (MEN). }\end{array}$ & $\stackrel{0}{\omega}$ & $\begin{array}{l}\text { Pos regulator of START. } \\
\text { Transcription factor for late G1 } \\
\text { genes. Forms complex with Swi4 to } \\
\text { form SBF, and with Mbp1 to form } \\
\text { MBF. Interacts with co-repressor } \\
\text { Whi5. }\end{array}$ \\
\hline 商 & $\begin{array}{l}\text { Pos regulator of M-G1. Activator of } \\
\text { the APC. Homolog of Cdc20. }\end{array}$ & $\frac{1}{2}$ & $\begin{array}{l}\text { Neg regulator of Metaphase-Anaphase. } \\
\text { SAC. Forms complex with Bub3 to } \\
\text { catalyze formation of Mad-Cdc20 MCC. }\end{array}$ & $\frac{m}{\frac{m}{3}}$ & $\begin{array}{l}\text { Neg regulator of START. RNA } \\
\text { binding protein that sequesters } \\
\text { CLN3 mRNA in cytoplasm. Paralog } \\
\text { of WHI4. }\end{array}$ \\
\hline$\overbrace{0}^{-}$ & $\begin{array}{l}\text { Pos regulator of G2-M. B-type } \\
\text { cyclin activator of Cdc28. Paralog of } \\
\text { CLB2. }\end{array}$ & $\frac{\Sigma}{2}$ & $\begin{array}{l}\text { Neg regulator of Metaphase-Anaphase. } \\
\text { SAC. Forms complex with Mad3/Cdc20 } \\
\text { to form MCC. Inhibits Anaphase } \\
\text { Promoting Complex (APC). }\end{array}$ & $\frac{d}{\frac{1}{2}}$ & $\begin{array}{l}\text { Neg regulator of START. Loss of } \\
\text { Whi } 4 \text { alone has no effect on cell } \\
\text { cycle,but does enhance cell cycle } \\
\text { phenotypes of whi3 mutants. } \\
\text { Paralog of WHI3. }\end{array}$ \\
\hline$\underset{\substack{\infty \\
0}}{\sim}$ & $\begin{array}{l}\text { Pos regulator of G2-M. B-type } \\
\text { cyclin activator of Cdc28. Paralog of } \\
\text { CLB1. }\end{array}$ & 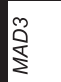 & $\begin{array}{l}\text { Neg regulator of Metaphase-Anaphase. } \\
\text { SAC. Forms complex with Mad2 and } \\
\text { Cdc20 to form MCC. Inhibits APC. }\end{array}$ & $\frac{10}{\frac{1}{x}}$ & $\begin{array}{l}\text { Neg regulator of START. Co- } \\
\text { repressor with SBF and MBF. } \\
\text { Homolog of Nrm1. }\end{array}$ \\
\hline$\frac{\infty_{\infty}^{\infty}}{\Delta}$ & $\begin{array}{l}\text { Pos regulator of DNA replication. } \\
\text { Also, involved in spindle dynamics } \\
\text { during M. B-type cyclin activator of } \\
\text { Cdc28. Paralog of CLB6. }\end{array}$ & $\frac{i}{2}$ & $\begin{array}{l}\text { Pos regulator of START. Transcription } \\
\text { factor forms complex with Swi6 and } \\
\text { partially redundant with Swi4-Swi6 } \\
\text { transcription factor SBF. }\end{array}$ & $\frac{1}{2}$ & $\begin{array}{l}\text { Pos regulator of START. Cytosolic } \\
\text { HSP70 protein. Forms co- } \\
\text { chaperone complex with Ssa1. } \\
\text { Involved in reversing sequestration } \\
\text { of CLN3 mRNAs by Whi3 and } \\
\text { stress-response. }\end{array}$ \\
\hline 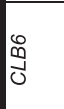 & $\begin{array}{l}\text { Pos regulator of DNA replication. } \\
\text { Also, involved in spindle dynamics } \\
\text { during M. B-type cyclin activator of } \\
\text { Cdc28. Paralog of CLB5. }\end{array}$ & $\sum_{\infty}^{\infty}$ & $\begin{array}{l}\text { Pos regulator of G1-S. Karyopherin } \\
\text { exportin involved in nuclear export of } \\
\text { Swi5 and Swi6. }\end{array}$ & $\frac{i}{\frac{1}{x}}$ & $\begin{array}{l}\text { Promotes proper timing of } \mathrm{M} \text { and } \\
\text { G1 transcription. Transcriptional co- } \\
\text { repressor with Mcm1. Paralog of } \\
\text { YOX1. }\end{array}$ \\
\hline$\sum_{U}^{5}$ & $\begin{array}{l}\text { Pos regulator of G1-S. G1 cyclin } \\
\text { activator of Cdc28. Paralog of } \\
\text { CLN2. }\end{array}$ & $\sum_{\substack{i \\
z}}^{\Gamma}$ & $\begin{array}{l}\text { Pos regulator of G1-S. Co-repressor } \\
\text { with MBF during S-phase. Homolog of } \\
\text { Whi5. }\end{array}$ & ত্র & $\begin{array}{l}\text { Promotes proper timing of } \mathrm{M} \text { and } \\
\mathrm{G} 1 \text { transcription. Transcriptional co- } \\
\text { repressor with Mcm1. Paralog of } \\
\text { YHP1. }\end{array}$ \\
\hline
\end{tabular}

Fig. 1 Genes used in this experiment. List of 36 cell cycle regulator genes used in the crosses.

our observed SL gene combinations are not listed on SGD. Hence, the overlap between the previously published SL interactions, and the combinations in our screen that exhibited synthetic lethality in at least four replicates is only $\sim 50 \%$. Dropping the threshold for likely SL interactions in our screen from four to three would have resulted in the identification of only one additional previously documented SL interaction (Ite1 $\Delta$ sic $1 \Delta$ ), while adding $13 \mathrm{SL}$ interactions that are not supported by the literature. Increasing the threshold to 5 would have excluded an additional $13 \mathrm{SL}$ interactions that have been previously observed.

As a check on these comparisons, we performed TA on at least one cross for all of the combinations listed in Fig. 4 except for one $(c d h 1 \Delta$ ssa1 $\Delta$ ) from which we failed to recover tetrads. Of the $13 \mathrm{SL}$ gene combinations that we observed for the first time in this study, 6 were not SL by TA $(b c k 2 \Delta c d h 1 \Delta$, bub2 $\Delta$ cdc55 $\Delta$, bub3 $\Delta$ swi $4 \Delta, c d c 55 \Delta$ nrm $1 \Delta, c d c 55 \Delta$ swi $\Delta \Delta$, and swi6 $\Delta$ whi3 $\Delta$ ). The other

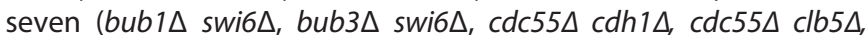

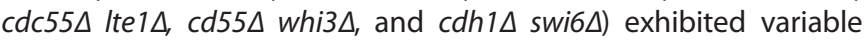

results or low spore viability regardless of genotype in at least one of the crosses, complicating the interpretation of the results. Of the 20 'documented' SL interactions that were not observed in our screen, 17/20 tested by TA were viable. The other three (Ite1 $\Delta$ sic $1 \Delta$, Ite $1 \Delta$ ydj $1 \Delta$, and $m s n 5 \Delta$ swi6 $\Delta$ ) varied by replicates or exhibited low spore viability overall, and thus remain ambiguous.

In summary, our screen identified 13 new potential SL interactions, but none of these were definitively validated by TA. Our screen failed to validate 20 previously published SL interactions. By TA, we determined that at least 17 of these are likely not SL. Of the 16 double-mutant combinations that were both 'documented' SL on SGD and 'likely' SL according to our screen, TA confirmed that nine combinations are indeed inviable. The other seven remain ambiguous.

Based on these comparisons, we re-classify the 58 gene combinations in Fig. 4 as 'high-confidence synthetic-lethal' combinations (shaded orange), 'high-confidence viable' doublemutants (shaded blue), and 'uncertain' (unshaded). Of those that 

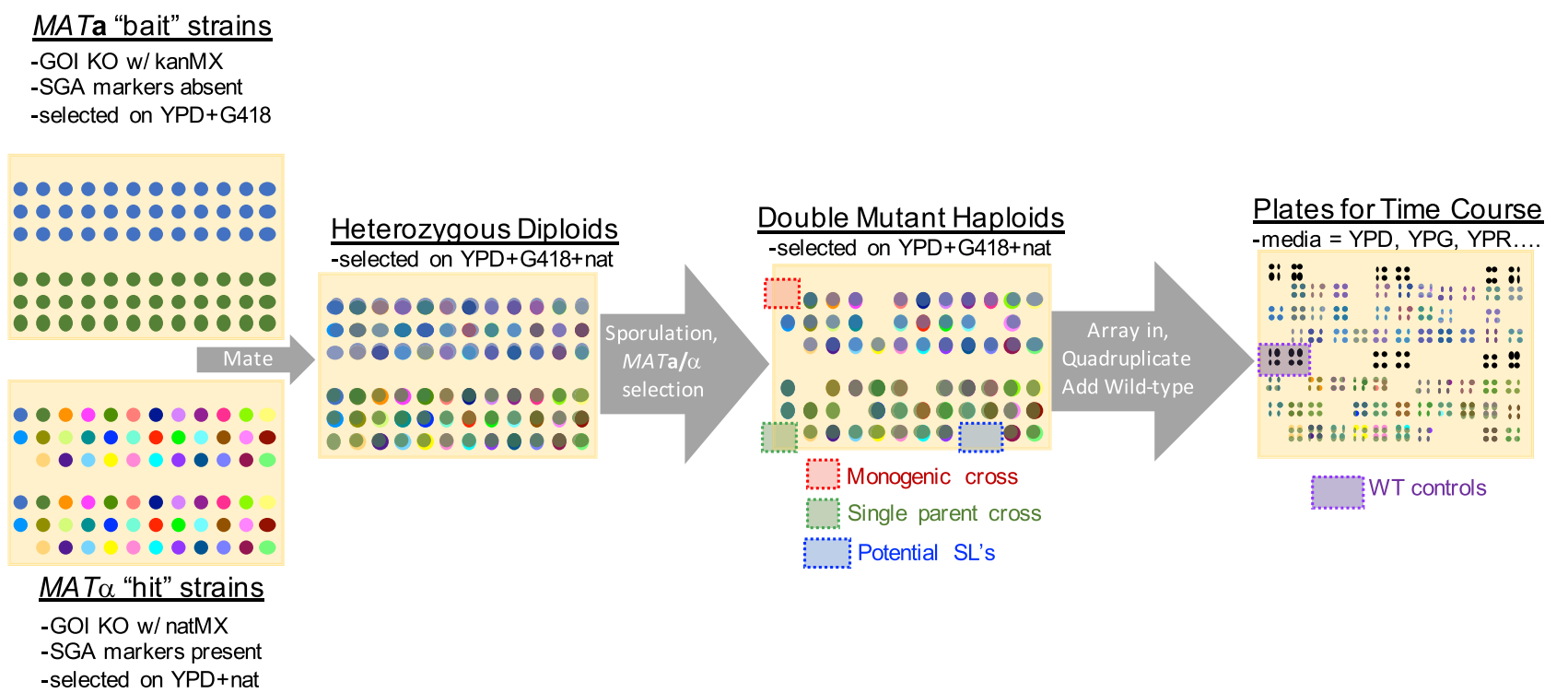

Fig. 2 Simplified representation of experimental workflow. Example of a single cross plate where two MATa 'bait' strains in which the gene of interest (GOI) was knocked out (KO) with a kanamycin resistant marker (kanMX, also confers resistance to G418) are each crossed to the 36 MATa 'hit' strains in which the gene of interest was knocked out with a nourseothricin-resistant marker (natMX). Heterozygous diploids were selected for on media containing both antibiotics, and then sporulated on standard sporulation media. The sporulated colonies were pinned onto a series of specialized SGA media that select for MATa and MAT $\alpha$ haploid progeny. Positions on the double mutant haploid plates that would have resulted in 'monogenic crosses' (where the same gene of interest was knocked out in both parents) or 'single parent crosses' (where one of the parent positions was empty) were monitored for potential false-negatives. The double mutant haploid progeny were used to identify synthetic lethal interactions and then pinned in quadruplicate on a fresh YPD plate. WT controls were added, and the resulting master plate was pinned onto six different media types for phenotyping. Phenotyping plates were imaged every $12 \mathrm{~h}$ to monitor growth rates.

remain uncertain, for five gene combinations which all include swi4 $\Delta$ or swi6 $\Delta$ (bub3 swi6 $\Delta$, clb5 swi6, msn5 swi4 $\Delta$, msn5 $\Delta$ swi6 $\Delta$, and swi4 $\Delta$ wi6 $\Delta$ ) additional replicates were attempted, but no tetrads were recovered.

Some of the variability observed between replicate tetrad analyses of the same genotype, as well as apparent meiotic defects, may be the result of chromosome loss. For instance, Bub1 and Bub3, which are involved in regulating the SAC and tension sensing in spindles ${ }^{32,33}$, exhibited unusual behavior in halo assays indicative of chromosome loss (see additional data).

Using our screen to refine a previously published model of the cell cycle

In addition to SGD, we compared our 'likely' SL interactions with those that were predicted by Kraikivski's model ${ }^{19}$. Of the 22 predicted SL gene combinations in Fig. 4, 10 are both documented and confirmed by our screen, two (bck2 swi6 $\Delta$ and $c \ln 3 \Delta$ swi $4 \Delta$ ) were documented but not observed by us, and one ( $c d h 1 \Delta$ swi6 $\Delta$ ) was observed by us but not documented on SGD. Nine predicted SL gene combinations were neither observed by us nor documented on SGD. We tested eight of these by TA and found six to be viable, while two (cdh1 1 lte $1 \Delta$ and cdh1 $1 \Delta$ ydj1 1 ) remain uncertain (Fig. 4). Five of these 'orphan' predictions involve $c d h 1 \Delta$, suggesting an overemphasis of Cdh1 activity in the model. We tested four of these combinations by TA and found that two were viable (cdh1 $\Delta \mathrm{clb} 5 \Delta$ and $\operatorname{cdh} 1 \Delta \operatorname{cln} 3 \Delta$ ), while two remain uncertain (cdh1 $1 \Delta$ lte $1 \Delta$ and $c d h 1 \Delta$ ydj1 $1 \Delta$ ). Six SL gene combinations that were both documented and observed by us were not analyzed in Kraikivski's model.

In summary, Kraikivski's model makes 37 predictions (22 SL + $15 \mathrm{~V})$ concerning the genetic interactions listed in Fig. 4. Of these predictions, 16 are consistent with our 'high-confidence' SL/V phenotypes, 7 are inconsistent (bolded in Fig. 4), and 14 are ambiguous. Hence, the accuracy of the published model is $\sim 50 \%$, comparable to the agreement between our screen and the literature.
The limited accuracy of the model's predictions is likely due to the fact that the parameter values in the model were estimated by fitting the model to 'documented' SL gene combinations that are themselves unreliable. To correct this problem, we have reparametrized the model in light of the 'high confidence' SL and viable (V) interactions (shaded orange and blue, respectively in Fig. 4), allowing for some flexibility for the uncertain interactions. In reparameterizing the model, we had two intentions: (a) to maximize the number of correctly explained mutant phenotypes in Fig. 4, and (b) to simulate correctly those mutant strains with well-characterized phenotypes that were previously explained by the model. Guided by these two criteria, we manually adjusted 13 parameter values in the model (Fig. S3), as follows:

- Because of the central roles played by SBF, MBF, and $\mathrm{Cln} 3$ in the START transition of the budding yeast cell cycle, we addressed our new results suggesting a viable phenotype for swi4 $\Delta \operatorname{cln} 3 \Delta$ double-mutant cells in opposition to previous reports that swi4 $\Delta \operatorname{cln} 3 \Delta$ is a $S L$ strain $^{25}$. To 'rescue' swi $\Delta \Delta \operatorname{cln} 3 \Delta$ cells, we significantly increased the activation of MBF (Swi6: Mbp1) by Bck2 (the only activator of MBF in the absence of Cln3), while simultaneously increasing the inactivation of MBF by $\mathrm{Clb} 2$ and decreasing slightly the activation of MBF by $\mathrm{Cln} 3$, in order to keep the level of MBF activity similar to that of the previous model, thus minimizing the perturbations to all other mutants that were previously explained by the model. Because $Y d j 1$ is a regulator of $C \ln 3$ activity, the phenotype of swi $\Delta$ ydj $1 \Delta$ agreed with new data too.

- The viability of swi6 $\Delta$ clb2 $\Delta$ suggests that Swi4 alone (without Swi6) can successfully initiate the START transition, and then the cell cycle can be completed without Clb2 (with Clb1 alone). To correctly simulate this mutant, we had to significantly increase the weight of Swi4 in the transcriptional regulation of the START transition.

- We also made adjustments to account for the five mutant strains involving $c d h 1 \Delta$ that our original model did not predict correctly (Table S6). In the model, cell division (upon exit from 
a

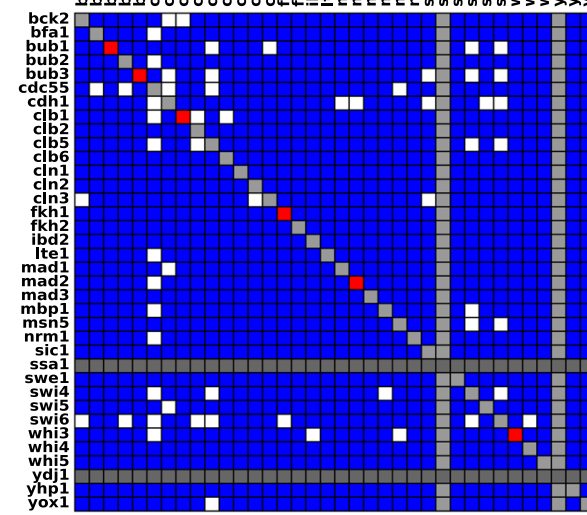

C

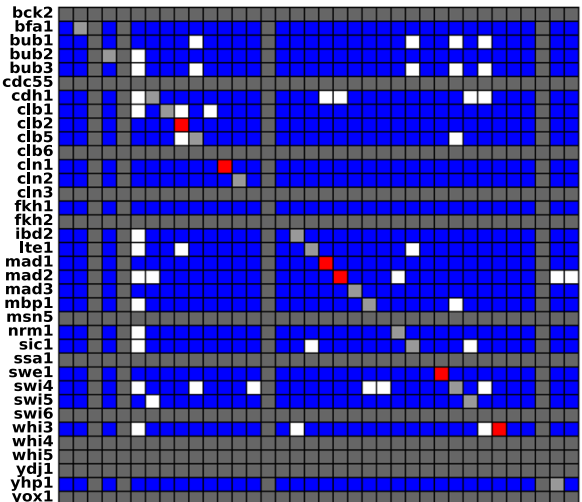

e

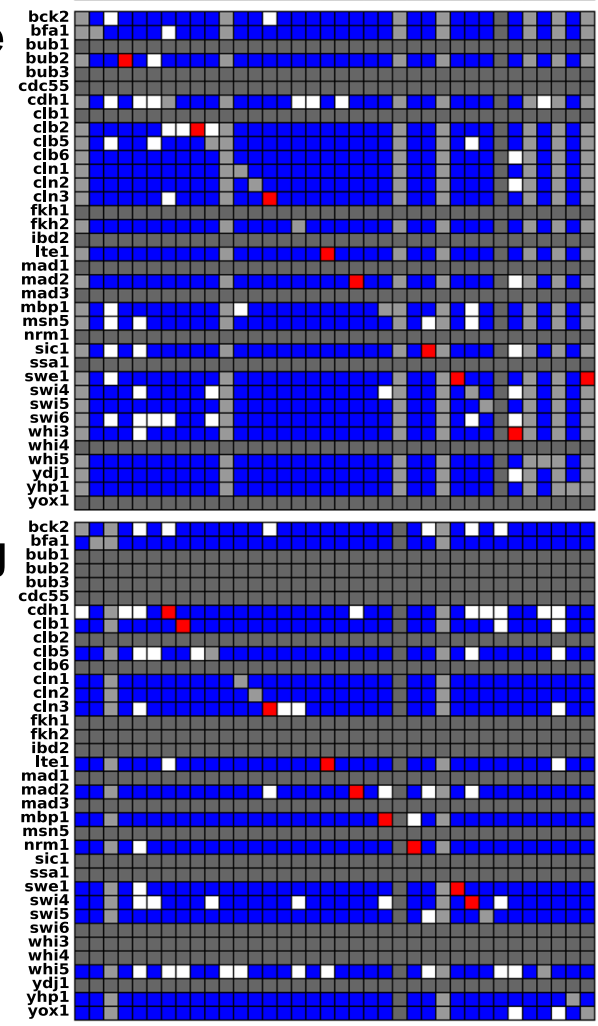

b

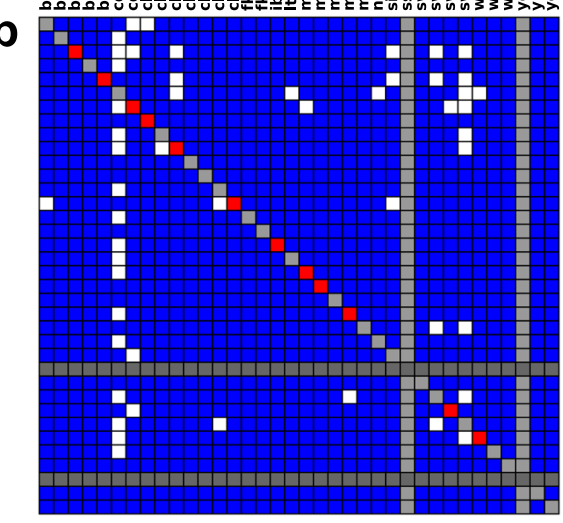

d

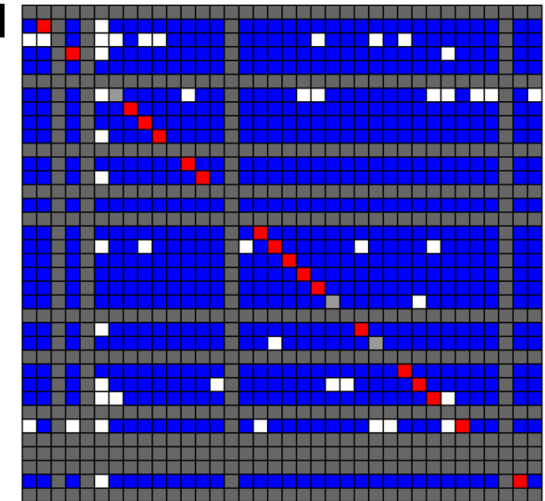

f

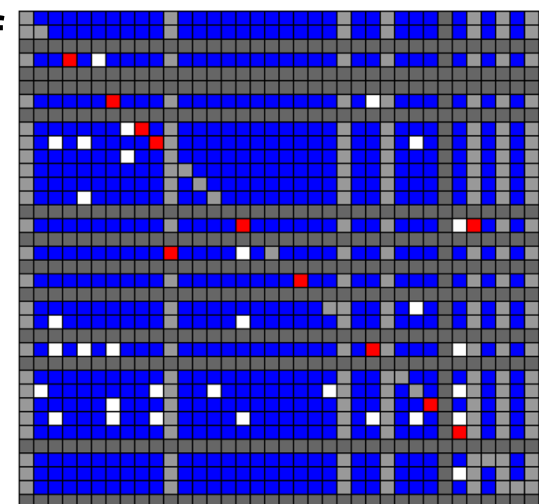

h

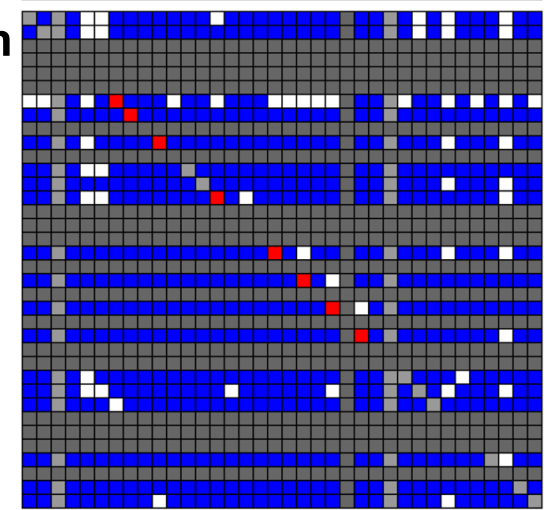

Key

口Zero Growth

a Non-Zero Growth

$\square$ Growth not expected Zero Growth

$\square$ Growth not expected Non-Zero Growth

$\square$ Parent not generated Or excluded mitosis) is determined by $\mathrm{Clb} 2$ activity dropping below a certain threshold, which is in turn governed by Cdh1 (involved in $\mathrm{Clb} 2$ degradation during telophase) and Sic1 (an inhibitor of Clb2-dependent kinase activity as cells return to G1). Hence, the inviability of cdh $1 \Delta$ sic $1 \Delta$ cells is the crucial mutant defining the cell-cycle exit threshold, and it was correctly predicted by the original model. In this double-mutant, Clb2dependent kinase activity is down-regulated in anaphase only by $\mathrm{Cdc} 20$-dependent degradation of $\mathrm{Clb2}$. (In reality, of course, $\mathrm{Clb} 2$ activity depends on many upstream regulators 
Fig. 3 Binary assessment of colony growth for double mutants in all four sets of crosses. Figures on the left were derived from MATa progeny. Figures on the right were derived from the sister MAT $\alpha$ progeny. MATa parents are organized along the $x$-axis and MAT $\alpha$ parents are organized along the $y$-axis alphabetically by the gene that was knocked out. Rows or columns shaded light gray indicate positions on the plate that should have been empty, because the parent was never generated. The diagonal in each heat map indicates a cross between two parents in which the same gene was knocked out. These should not result in growth under selection. Red cells indicate unexpected growth and are an indication of the false negative rate. Rows and columns shaded dark gray indicate parents that were never generated or were excluded from the analysis, because at least one-third of the progeny resulting from that parent failed to grow. Duplicates of the same gene/ marker combination within the same cross are not shown. Total number of crosses (excluding monogenic) $=7350$. a, b Cross 1 . c, d Cross 2 . e, f Cross 3. g, h Cross 4.

— such as Ydj1, Clb5, Ssa1, Cln3, and Swi6-that affect cell mass at division.) Our new assessment of SL interactions allows for better 'tuning' of the parameters that govern $\mathrm{Clb} 2$ regulation by $\mathrm{Cdh} 1, \mathrm{Cdc} 20$, and Sic1. In addition, when originally constructing and parametrizing our model, we did not have many lte1 $\Delta$ mutant strains to constrain Lte1-related parameters, so we adjusted parameters to correctly explain lte $1 \Delta$ mutants.

The revised model is provided as $\mathrm{SBML}^{34}$ file in the Figshare dataset (see Data availability). The 2015 model is robust enough that the dynamics of WT and most mutants is the same for both models. Only some mutants show the different phenotype and distinct time course of cell cycle variables (Fig. S4). We have updated the list of mutant phenotypes predicted by the revised model and compared it with the predictions of the 2015 model (Fig. S5). We simulated the phenotype of 38 new mutants included in our experimental design. The predicted viability of 12 mutants changed as a result of the parameter adjustment and 15 mutants exhibit inconsistencies between the predicted and observed viability 321 simulated mutants. Most of these discrepancies involve specific types of mutations (multiple gene copies, GALinducible genes, and destruction box deletions) that are known to be less reproducible than simple deletions. In addition, the predicted viability of these mutants can be very sensitive to the parameters introduced in the model to capture the quantitative effects of these mutations.

Predictions of the newly parametrized model are given in the last column of Fig. 4. Our expertize in cell cycle regulation and mutant behavior allowed us to make these parameter adjustments manually; however, computational algorithms for reparameterization may be required if a larger number of novel mutant phenotypes becomes available in the future.

\section{Inherent limitations of synthetic lethality screens}

The SGA process relies on efficient production of double mutant haploid progeny from crosses. Mating defects, low sporulation efficiencies, meiotic defects causing poor spore viability, poor spore germination, or technical problems with the pinning process can prevent the transfer of double mutant cells during haploid selection, resulting in false positives (i.e., poorer growth than there should $b^{29,35}$ ). Genetic interactions resulting in reduced fitness are also subject to significant selection for genetic mishaps that improve fitness, resulting in false negatives (i.e., better growth than there should be ${ }^{29,35}$ ). Genetic mishaps resulting in false negatives can include spontaneous mutation to introduce a suppressor mutation ${ }^{36}$, or disomy. Disomy can result from chromosome nondisjunction during sporulation, or gene conversion resulting in escape of heterozygous diploids from haploid selection ${ }^{35,37}$. False negatives can also result from contamination from outside sources or cross-contamination during replica-pinning.

Following the presence or absence of colonies throughout the SGA process, we found that all crosses produced diploids (see Additional data). Therefore, failure to mate did not produce any false positives. False positives can also result from inefficient pinning or systematic problems with the parents resulting in overall low viability. Parent lines that resulted in fewer than 12/36 viable haploid progeny were excluded from the analysis, but some false positives likely persisted. For instance, seven of the SL gene combinations observed in our screen involved $c d c 55 \Delta$, which was problematic in most genetic contexts due to inconsistent pinning (cells were very dry and clumpy and did not adhere well to pins). By TA, we identified 6 out of 29 SL gene combinations observed in our screen to be definitive false positives.

Our experimental design makes it possible to get rough estimates of false negative rates by monitoring positions on each plate that should have been empty for growth. We designed our screen such that 'hit' strains were arrayed the same way (alphabetically by the gene knocked out) for every cross, leaving empty spaces for any parent that was not generated (Fig. 2). In this way, some positions in the cross had only one parent crossed to an empty position ('single parent' in Fig. 2), and some positions had two parents that were mutant for the same allele ('monogenic cross' in Fig. 2). Neither of these 'crosses' should result in colonies during the final round of double-mutant haploid selection, as they will not contain both of the antibiotic resistance markers. Colonies at 'single parent' or 'monogenic cross' positions are indicative of a false-negative event (red cells in Fig. 3).

To estimate the contribution of contamination to false negatives, we identified colonies in empty plate positions. All plates were devoid of contaminating colonies in empty positions (Table S3). In positions containing only one parent strain, only $3 /$ 570 positions on the haploid progeny plates had any contaminating colonies (Table S3). Therefore, contamination is a negligible contributor to observed false negatives.

To identify false negatives arising from genetic mishaps, we identified colonies produced by crosses between two strains carrying deletions of the same gene. Out of 202, 77 monogenic crosses resulted in progeny on the final haploid selection plates (Table S3), indicating a coarsely estimated false-negative rate of $38 \%$.

These false-negative events occurred more frequently for MATaATcurred more MATa progeny (Table S3). This is to be expected, because MATa progeny can escape selection for MATaATogeny can escape selection for expecteSTE3pr-LEU2 and leu2 $\triangle 0$, but gene conversion cannot occur between STE2pr-SpHIS3 (Schizosaccharomyces pombe orthologue) and his $3 \Delta 1$ to allow MATa cells to escape MATa selection ${ }^{29,35}$. If MATa progeny persist through the MATa selection due to gene conversion, they can mate with the neighboring MATa progeny producing diploids that are heterozygous for both markers.

Although few SGA or E-MAP studies report them, it is wellestablished that these screens have high, but variable, falsenegative and false-positive rates from $17 \%$ to $70 \%^{30,37-40}$ and $5 \%$ to $90 \%{ }^{39,41-44}$, respectively. The false-positive and -negative rates observed in our study are thus in the normal range for large genetic screens.

Quantifying fitness and genetic interactions across six media types As the most extreme genetic interaction, synthetic lethality has a powerful influence on models of cell-cycle regulating genes. 


\begin{tabular}{|c|c|c|c|c|c|c|c|c|}
\hline \multirow[t]{2}{*}{ Gene 1} & \multirow[t]{2}{*}{ Gene 2} & \multicolumn{2}{|c|}{ Documented } & \multicolumn{3}{|c|}{ Observed } & \multirow{2}{*}{$\begin{array}{c}\text { Kraikivski } \\
\text { Model }\end{array}$} & \multirow{2}{*}{$\begin{array}{c}\text { New } \\
\text { Model }\end{array}$} \\
\hline & & Method & Refs & SL & SL & TA & & \\
\hline BCK2 & $\mathrm{CDH} 1$ & & & $\mathrm{Y}$ & $5 / 14$ & $\mathrm{~V}, \mathrm{~V}, \mathrm{~V}$ & $\mathrm{~V}$ & V \\
\hline BCK2 & CLN3 & Manual & $34,86-90$ & $Y$ & $6 / 12$ & $\mathrm{SL}^{*}, \mathrm{SL}, \mathrm{RV}$ & $\mathrm{SL}$ & $\mathrm{SL}$ \\
\hline BCK2 & SWI4 & HTP & 91 & & $2 / 16$ & $\mathrm{~V}, \mathrm{~V}, \mathrm{~V}$ & $\mathrm{~V}$ & $\mathrm{~V}$ \\
\hline BCK2 & SWI6 & Manual & 34 & & $3 / 6$ & $\mathrm{~V}, \mathrm{~V}, \mathrm{~V}$ & $S L$ & $S L$ \\
\hline BFA1 & BUB3 & HTP & 40 & & $1 / 10$ & $\mathrm{~V}$ & & \\
\hline BFA1 & LTE1 & Manual & 92 & & $0 / 16$ & $\mathrm{~V}, \mathrm{~V}, \mathrm{~V}$ & $\mathrm{~V}$ & $\mathrm{~V}$ \\
\hline BUB1 & CLB5 & Manual & 32 & $Y$ & $6 / 8$ & $\mathrm{SL}^{*}, \mathrm{SL}^{*}$ & & \\
\hline BUB1 & SIC1 & Manual & 93 & $\mathrm{Y}$ & $5 / 8$ & $\mathrm{~V}, \mathrm{SL}, \mathrm{MD}, \mathrm{V}$ & & \\
\hline BUB1 & SWI6 & & & $Y$ & $6 / 10$ & $\mathrm{RV}^{*}, \mathrm{RV}^{*}$ & & \\
\hline BUB2 & CDC55 & & & $Y$ & $4 / 8$ & $\mathrm{~V}, \mathrm{~V}$ & $\mathrm{~V}$ & $\mathrm{~V}$ \\
\hline BUB3 & $\mathrm{CDH} 1$ & HTP & 64 & & $2 / 10$ & $\mathrm{~V}$ & & \\
\hline BUB3 & CLB1 & HTP & 64 & & $0 / 8$ & $\mathrm{~V}$ & & \\
\hline BUB3 & CLB5 & HTP/manual & 32,64 & $Y$ & $5 / 10$ & $\mathrm{SL}$ & & \\
\hline BUB3 & SIC1 & HTP & 64 & $\mathrm{Y}$ & $5 / 8$ & SL & & \\
\hline BUB3 & SWI4 & & & $\mathrm{Y}$ & $7 / 12$ & $\mathrm{~V}, \mathrm{~V}$ & & \\
\hline BUB3 & SWI6 & & & $Y$ & $4 / 10$ & $\mathrm{~V}, \mathrm{SL}$ & & \\
\hline BUB3 & YDJ1 & HTP & 64 & & $0 / 2$ & $\mathrm{~V}$ & & \\
\hline CDC55 & $\mathrm{CDH} 1$ & & & $\mathrm{Y}$ & $6 / 10$ & $\mathrm{SL}^{*}, \mathrm{~V}, \mathrm{MD}$ & $\mathrm{V}$ & $\mathrm{V}$ \\
\hline CDC55 & CLB5 & & & $Y$ & $7 / 10$ & SL, RV & $\mathrm{V}$ & SL \\
\hline CDC55 & LTE1 & & & $Y$ & $5 / 10$ & $\mathrm{SL}, \mathrm{RV}$ & $\mathrm{V}$ & $\mathrm{V}$ \\
\hline CDC55 & MSN5 & Manual & 94 & & $1 / 6$ & $\mathrm{~V}, \mathrm{~V}, \mathrm{RV}^{*}$ & & \\
\hline CDC55 & NRM1 & & & $\mathrm{Y}$ & $5 / 8$ & $\mathrm{~V}, \mathrm{~V}$ & & \\
\hline CDC55 & SWI4 & & & $Y$ & $4 / 12$ & $\mathrm{~V}, \mathrm{~V}, \mathrm{MD}$ & $\mathrm{V}$ & $\mathrm{V}$ \\
\hline CDC55 & WHI3 & & & $Y$ & $4 / 8$ & $\mathrm{MD}, \mathrm{MD}$ & & \\
\hline $\mathrm{CDH} 1$ & CLB5 & & & & $0 / 16$ & V & SL & $\mathrm{V}$ \\
\hline $\mathrm{CDH} 1$ & CLN3 & & & & $2 / 16$ & $\mathrm{~V}$ & SL & V \\
\hline $\mathrm{CDH} 1$ & LTE1 & & & & $2 / 16$ & $\mathrm{~V}, \mathrm{RV}, \mathrm{MD}$ & $S L$ & V \\
\hline $\mathrm{CDH} 1$ & MAD1 & HTP & 64 & $\mathrm{Y}$ & $4 / 12$ & $\mathrm{RV}, \mathrm{V}$ & & \\
\hline $\mathrm{CDH} 1$ & MAD2 & HTP & 64 & $\mathrm{Y}$ & $6 / 16$ & $\mathrm{~V}, \mathrm{MD}, \mathrm{V}$ & $\mathrm{SL}$ & SL \\
\hline $\mathrm{CDH} 1$ & SIC1 & HTP/manual & 93,95 & $Y$ & $4 / 14$ & SL & $S L$ & $\mathrm{SL}$ \\
\hline $\mathrm{CDH} 1$ & SSA1 & & & & $0 / 2$ & & $S L$ & $\mathrm{~V}$ \\
\hline $\mathrm{CDH} 1$ & SWI4 & HTP & 91 & & $0 / 18$ & $\mathrm{~V}, \mathrm{~V}, \mathrm{~V}$ & $\mathrm{~V}$ & $\mathrm{~V}$ \\
\hline $\mathrm{CDH} 1$ & SWI5 & Manual & 96 & $Y$ & $10 / 16$ & $\mathrm{SL}, \mathrm{RV}^{*}$ & $S L$ & $\mathrm{SL}$ \\
\hline $\mathrm{CDH} 1$ & SWI6 & & & $\bar{Y}$ & $7 / 12$ & $\mathrm{SL}, \mathrm{V}, \mathrm{MD}, \mathrm{MD}$ & $\mathrm{SL}$ & $\mathrm{SL}$ \\
\hline $\mathrm{CDH} 1$ & WHI5 & HTP & 91 & $Y$ & $4 / 18$ & $\mathrm{~V}, \mathrm{RV}^{*}$ & SL & $\mathrm{SL}$ \\
\hline $\mathrm{CDH} 1$ & YDJ1 & & & & $2 / 4$ & $\mathrm{SL}, \mathrm{V}, \mathrm{RV}$ & $S L$ & $\mathrm{~V}$ \\
\hline CLB1 & CLB2 & Manual & $97-100$ & $Y$ & $4 / 12$ & $\mathrm{SL}, \mathrm{SL}$ & $S L$ & $\mathrm{SL}$ \\
\hline CLB2 & MAD2 & HTP & 64 & & $0 / 14$ & $\mathrm{~V}$ & $\mathrm{~V}$ & $\mathrm{~V}$ \\
\hline CLB2 & SWI6 & & & & $1 / 10$ & $\mathrm{~V}$ & SL & V \\
\hline CLB2 & SIC1 & Manual & 101,102 & & $0 / 12$ & $\mathrm{~V}, \mathrm{~V}$ & $\mathrm{~V}$ & V \\
\hline CLB5 & SIC1 & Manual & 101,102 & & $0 / 14$ & $\mathrm{~V}, \mathrm{~V}, \mathrm{RV}$ & $\mathrm{V}$ & $\mathrm{V}$ \\
\hline CLB5 & SWI4 & Manual & 103 & $\mathrm{Y}$ & $10 / 18$ & $\mathrm{RV}^{*}, \mathrm{SL}$ & $S L$ & $\mathrm{SL}$ \\
\hline CLB5 & SWI6 & Manual & 103 & $Y$ & $4 / 12$ & $\mathrm{~V}, \mathrm{~V}, \mathrm{SL}$ & SL & $\mathrm{SL}$ \\
\hline CLN3 & SWI4 & HTP, manual & 34,91 & & $2 / 18$ & $\mathrm{~V}, \mathrm{~V}, \mathrm{~V}, \mathrm{~V}$ & SL & $\mathrm{V}$ \\
\hline LTE1 & MAD2 & & & & $1 / 16$ & $\mathrm{~V}$ & SL & V \\
\hline LTE1 & SIC1 & HTP & 93 & & $3 / 14$ & $\mathrm{~V}^{*}, \mathrm{SL}$ & $\mathrm{V}$ & V \\
\hline LTE1 & SWI6 & & & & $1 / 12$ & V & SL & V \\
\hline LTE1 & YDJ1 & HTP & 104 & & $2 / 4$ & $\mathrm{SL}, \mathrm{SL}, \mathrm{V}$ & $\mathrm{V}$ & $\mathrm{V}$ \\
\hline MAD1 & SIC1 & HTP & 64 & & $0 / 10$ & $\mathrm{~V}$ & & \\
\hline MAD2 & SIC1 & HTP & 64 & & $1 / 14$ & $\mathrm{~V}, \mathrm{~V}$ & V & V \\
\hline MAD2 & WHI5 & HTP & 64 & & $1 / 18$ & $\mathrm{~V}$ & V & V \\
\hline MAD3 & SIC1 & HTP & 64 & & $0 / 10$ & $\mathrm{~V}$ & & \\
\hline MBP1 & SWI4 & Manual & 87,105 & $Y$ & $15 / 20$ & $S L$ & SL & $\mathrm{SL}$ \\
\hline MSN5 & SWI4 & Manual & 106,107 & $Y$ & $4 / 8$ & $\mathrm{~V}$ & & \\
\hline MSN5 & SWI6 & Manual & 106,107 & & $2 / 4$ & $\mathrm{RV}, \mathrm{V}$ & & \\
\hline SWI4 & SWI6 & Manual & $34,108,109$ & $Y$ & $12 / 14$ & $\mathrm{RV}^{*}, \mathrm{~V}^{*}, \mathrm{MD}$ & $\mathrm{SL}$ & $\mathrm{SL}$ \\
\hline SWI4 & YDJ1 & & & & $2 / 6$ & $\mathrm{~V}$ & SL & $\mathrm{V}$ \\
\hline SWI6 & WHI3 & & & $\mathrm{Y}$ & $4 / 10$ & $\mathrm{~V}, \mathrm{~V}$ & & \\
\hline \multicolumn{2}{|c|}{ Total \# SL } & \multicolumn{2}{|l|}{36} & \multicolumn{3}{|c|}{29 (6 viable by TA) } & 22 & 13 \\
\hline
\end{tabular}

Fig. 4 Curation of synthetic lethal interactions. For previously documented SL interactions, it is noted whether they were manually curated or derived from a high-throughput (HTP) screen. The 'SL score' highlights the number of crosses in our screen that support synthetic lethality out of the total number of replicates. Double mutant combinations are reported as synthetic lethal (SL), viable (V), or having reduced viability (RV). More than one reported TA result indicates that the TA was repeated for two or more crosses. Results marked with an asterisk are lower confidence due to poor spore viability. Cases where spore viability was too low to determine synthetic lethality are marked MD to indicate a possible meiotic defect. Rows shaded orange mark likely synthetic lethal interactions. Rows shaded blue mark likely viable interactions. Instances where the model does not support the 'high confidence' interactions are bolded. 
a

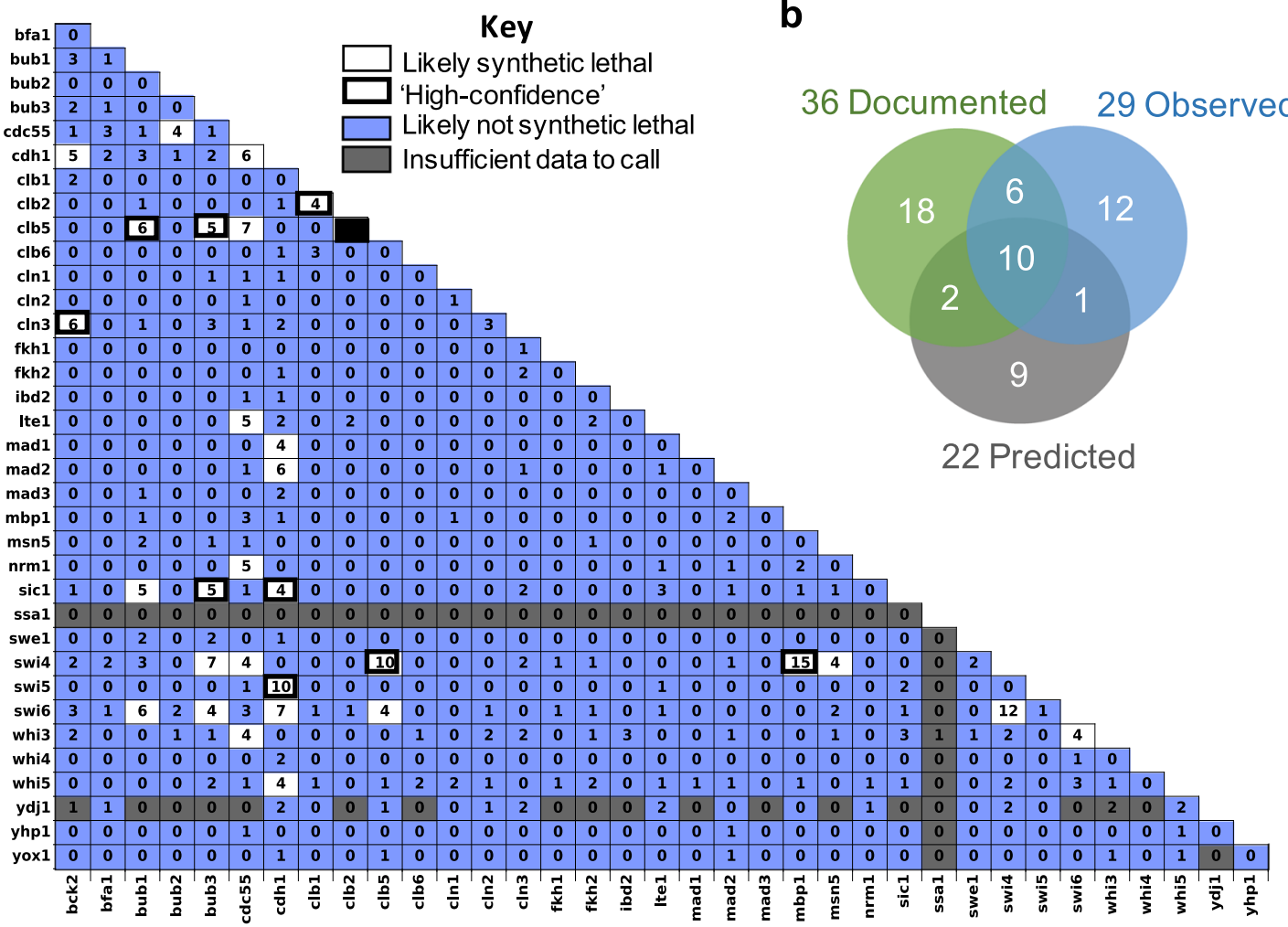

Fig. 5 Likely synthetic lethal interactions determined by compiling data from all crosses. a Table documenting how many crosses supported synthetic lethality (no growth of the double mutant progeny). Synthetic lethal interactions that we designate as 'high-confidence' in Fig. 4 are outlined in black. b Venn-Diagram comparing observed SL interactions with those that have been previously documented and/or predicted by the Kraikivski model. Note: $\mathrm{clb} 2 \Delta \mathrm{clb} 5 \Delta$ is excluded as these genes are linked.

However, due to the limitations of synthetic lethality screens more accurate models call for more nuanced phenotypic markers.

To identify interactions between the 36 genes that do not result in synthetic lethality, we monitored the growth rate of the viable double mutants over a time course. Each mutant was assigned a fitness score according to how the growth rate compared with wild-type controls on the same plate. Using this approach, we identified $\sim 100$ gene combinations that were not SL but had fitness scores more than six standard deviations below wild-type under normal growth conditions (Fig. 6).

More importantly, by comparing fitness scores of the double mutant progeny with those of their single mutant parents, we calculated genetic interaction (GI) scores for all viable mutants. Gl scores $^{45-47}$ are a function of the parent and progeny fitness and illustrate the direction (positive or negative) and the magnitude of the interaction for each of the $\sim 600$ viable gene combinations. Non-zero Gl scores indicate a possible epistatic relationship. Negative Gl scores suggest that the genes involved may have redundant functions, while positive Gl scores indicate that one mutation may have a rescuing effect over the other.

As with synthetic lethality, we observed a considerable amount of variability in fitness scores and Gl scores for mutants of the same genotype in different crosses (biological replicates, Fig. 6). To identify trends within the variability, Gl scores for a given genotype were sorted into different bins, and the bin that contained the largest number of biological replicates was used to determine a consensus Gl score which is represented in Fig. 7 and Fig. 8. From the distribution of overall GI scores for a given media, we flagged those with a consensus score at the extreme positive and negative ends. Those gene combinations with consensus $\mathrm{Gl}$ scores in the top or bottom $5 \%$ of all Gl scores are reported in Fig. 8.

To further identify Gls among our set of cell cycle regulator genes that may not be apparent under standard growth conditions, we also calculated fitness and Gl scores for all double mutant progeny and single mutant parents in the presence of two different carbon sources and in the presence of three checkpoint activating drugs (Figs. S6-S10).

YPDextrose served as a control (mass doubling time $\sim 100 \mathrm{~min}$ ). Mass doubling times are longer on YPGalactose $(\sim 150 \mathrm{~min})$ and even longer on YPRaffinose $(\sim 200 \mathrm{~min})^{48}$. Slower growth rates can enable positive regulators to build up such that mutants which would normally grow very slowly due to the stochasticity of cell cycle transitions can exhibit some level of rescue on YPG or YPR $^{23,49}$

The distribution of $\mathrm{Gl}$ scores that we observed was comparable for YPD and YPG, but the GI scores occupied a narrower range for mutants grown on YPR (Fig. 7), suggesting that the very slow growth rate provided by YPR might allow the growth of mutants that have more extreme phenotypes on YPD to normalize on YPR.

The drugs Benomyl (Ben), camptothecin (CPT), and hydroxyurea (HU) activate checkpoints ${ }^{50-60}$. Mutants defective in these checkpoints will rush through the cell cycle and accumulate genetic/chromosome defects leading to slower growth due to decreased viability. We expect known checkpoint mutants to exhibit reduced fitness under these conditions, but interactions with other cell cycle regulators (including other checkpoint genes) can enhance or suppress the checkpoint defects ${ }^{32,61-67}$.

In several cases, gene combinations that had a Gl score within the normal distribution on YPD, showed a much more extreme Gl on one or more of the other five media types (Fig. 8). For instance, 
a

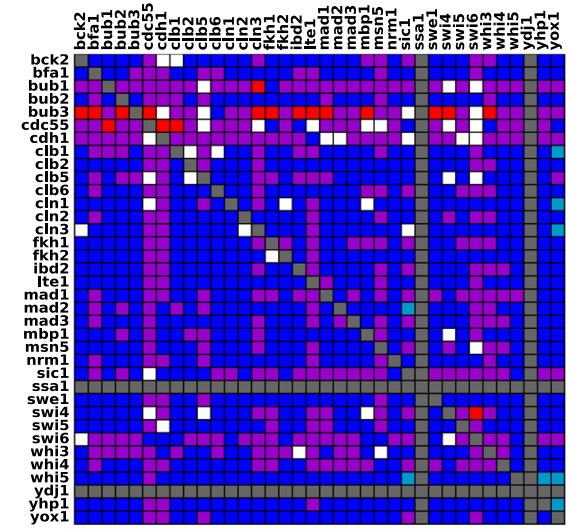

C

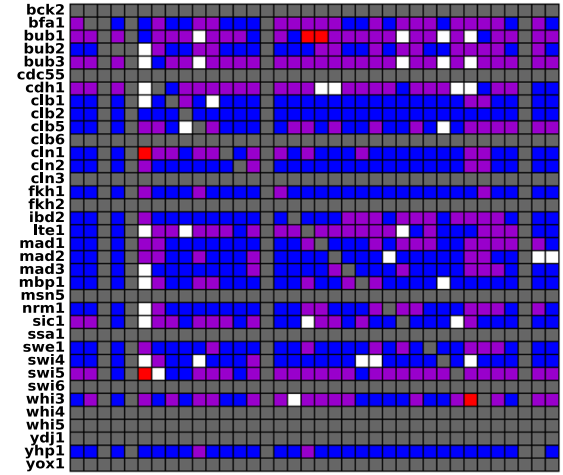

e

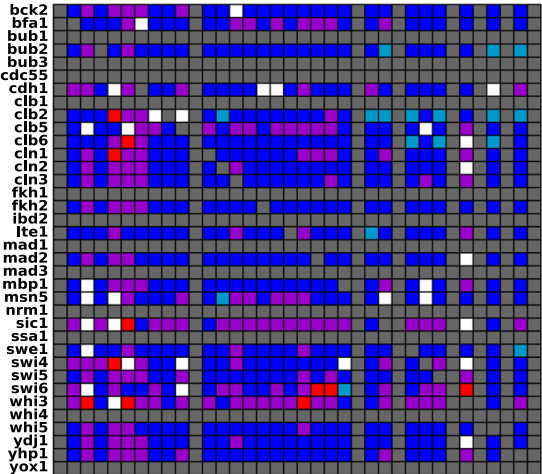

g

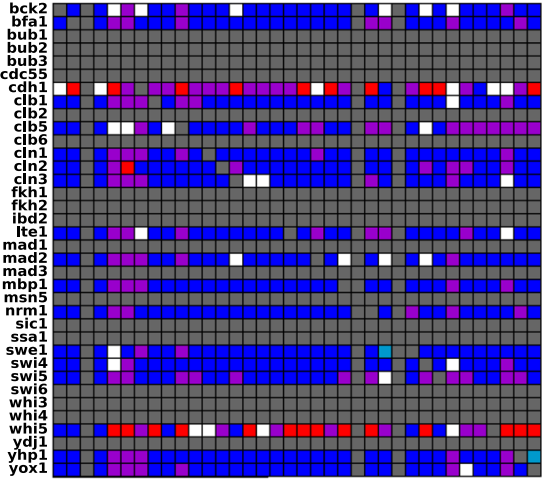

b
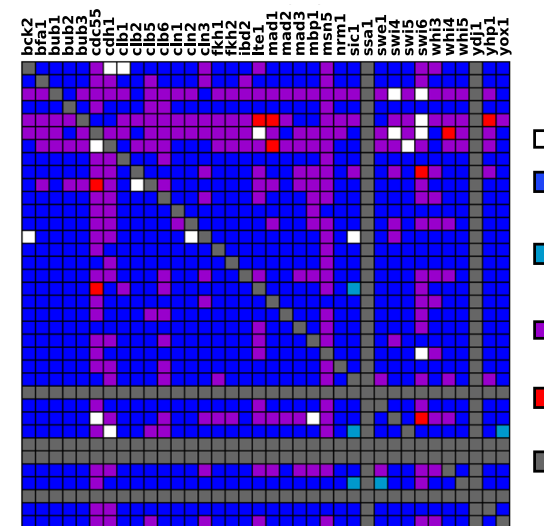

d

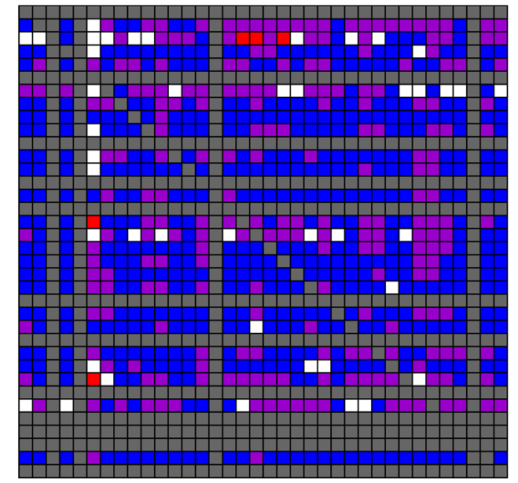

f

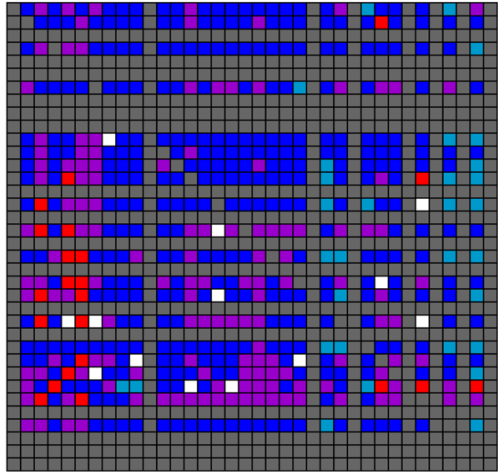

h

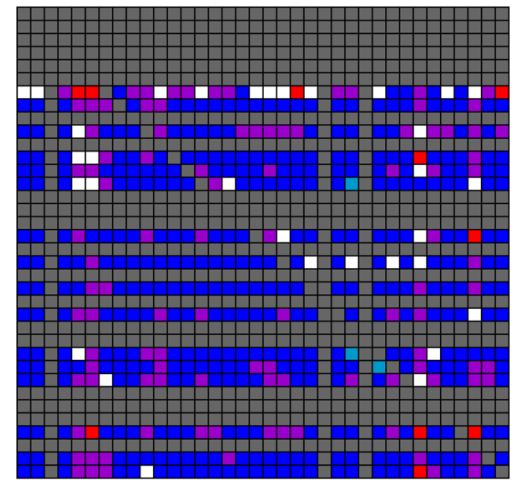

Key

$\square$ Zero Growth

Within 2 standard

deviations of WT

2-6 standard deviations greater than WT

2-6 standard deviations less than WT

$>6$ standard deviations less than WT

$\square$ Data missing or excluded

Fig. 6 Comparison of fitness scores for double mutants in all four sets of crosses on YPD media. Figures on the left were derived from MATa progeny. Figures on the right were derived from the sister MAT $\alpha$ progeny. MATa parents are organized along the $x$-axis and MAT $\alpha$ parents are organized along the $y$-axis alphabetically by the gene that was knocked out. Rows and columns shaded dark gray indicate crosses that were not performed or were excluded from the analysis. a, b Cross 1. c, d Cross 2. e, $\mathbf{f}$ Cross 3 . g, $\mathbf{h}$ Cross 4 . Fitness heatmaps for the remaining five media types are available is Figs. S3-S7. Duplicates of the same gene/marker combination within the same cross are not shown.

the Gl score for $f k h 1 \Delta f k h 2 \Delta$ on YPD was negative, but not remarkably so. However, on YPD + Ben and YPD $+C P T$, the consensus Gl scores for fkh1 $1 \mathrm{fkh} 2 \Delta$ were in the lower $5 \%$ and $2.5 \%$, respectively. Fkh1 and Fkh2 both promote the transition from $G 2$ to $M$, so the double mutant is likely to cause stalling at G2. Ben prevents spindle assembly while activating the spindle assembly checkpoint, so that cells move forward to $M$ phase despite not properly forming a mitotic spindle. CPT causes DNA 

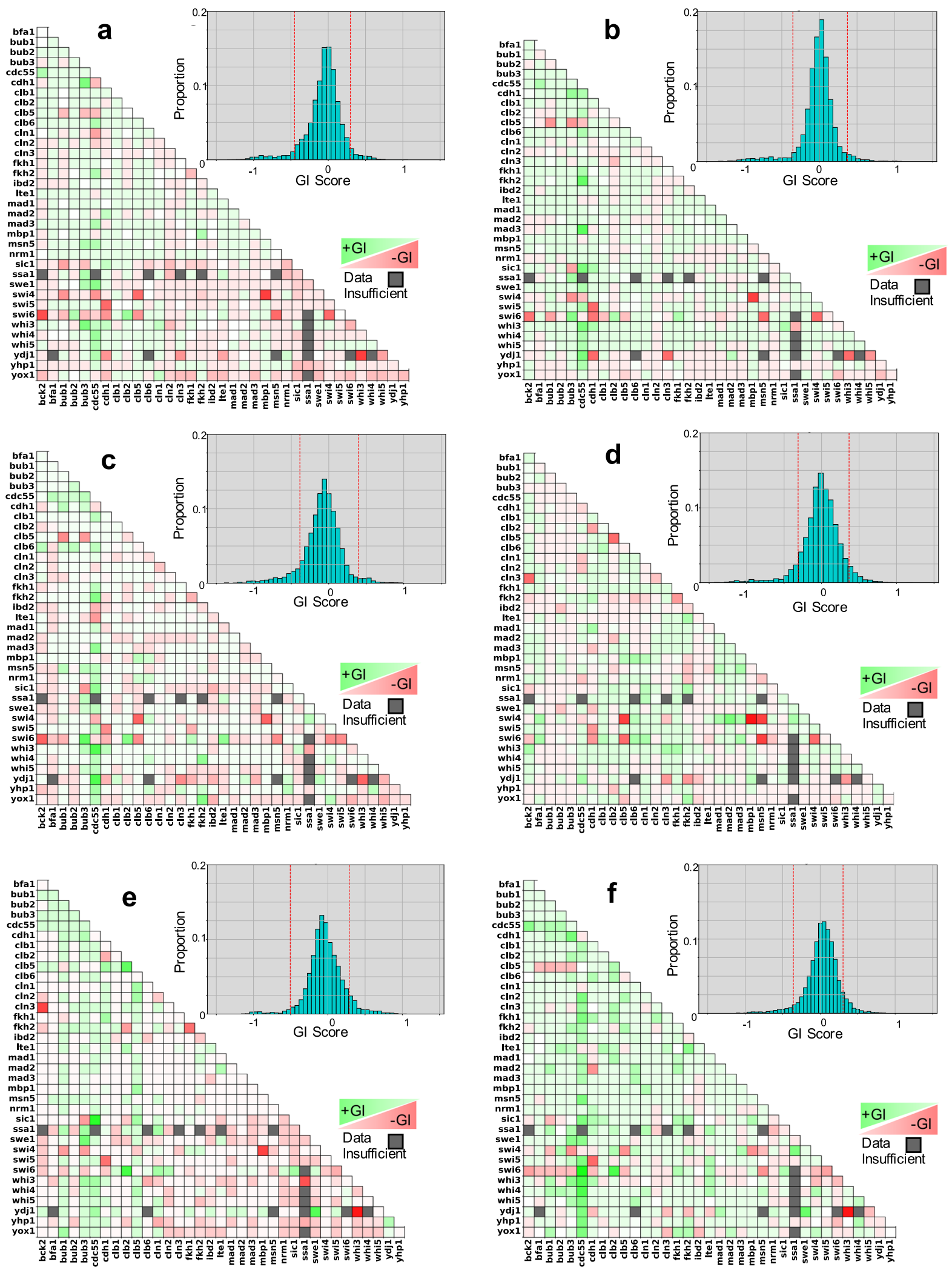

Fig. 7 Genetic interaction scores on each media type determined by compiling data from all crosses. Heat maps show the distribution of binned genetic interaction (Gl) scores for each mutant combination. Brighter green and darker red squares correspond to higher positive and lower negative GI scores, respectively. Gray squares denote gene combinations for which three or fewer crosses were generated. Histograms show the overall distribution of GI scores for each media type. The dotted red lines distinguish the lower and upper 5\% of interactions. a YPD, b YPD + raffinose, c YPD + galactose, d YPD + benomyl, e YPD + camptothecin, f YPD + hydroxyurea. 


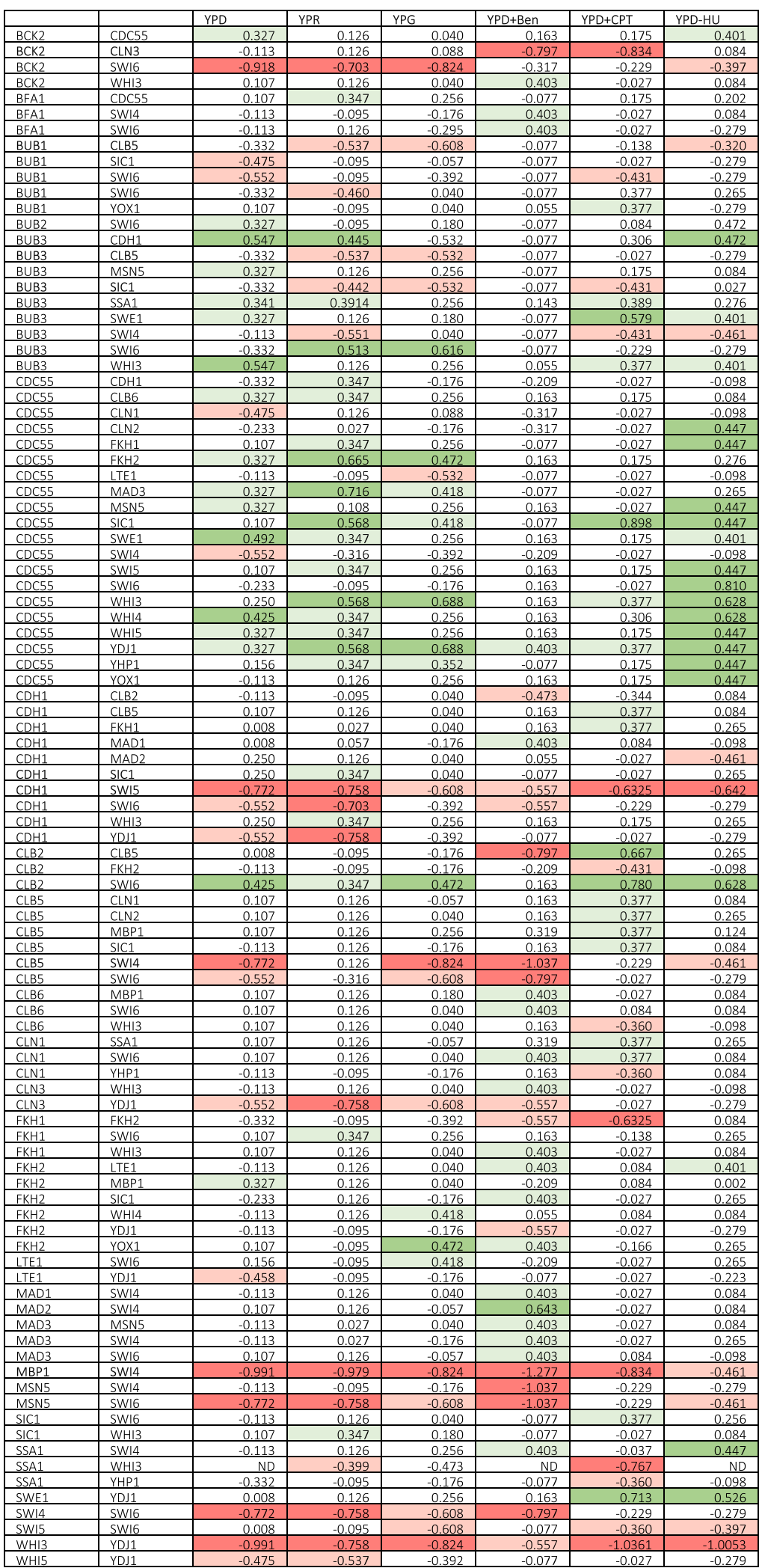

Fig. 8 Genetic interaction score outliers for each media type. Consensus genetic interaction (GI) scores are reported for each gene combination that had a score in the top or bottom $5 \%$ of overall $\mathrm{Gl}$ scores for one or more media types. The number reported reflects the midpoint of the bin occupied by the largest number of biological replicates. Red and green shading highlights negative and positive scores, respectively. The top $2.5 \%$ and $5 \%$ are shaded light and dark green, respectively. The bottom $2.5 \%$ and $5 \%$ are shaded light and dark red, respectively. Interactions which we determined to be very likely synthetic lethal are shown in bold. 'ND' indicates Gl scores that were not determined. 
damage during $M$ phase. So, cells that make it to $M$ phase in an $f k h 1 \Delta f k h 2 \Delta$ mutant would likely arrest in the presence of Ben or CPT, thus exacerbating the mutant phenotype.

Relative Gl scores for a family of gene combinations also reflect the role of those genes within the cell-cycle regulatory network. For instance, Bub1 and Bub3 function along with Mad1, Mad2, and Mad3 to arrest cells in metaphase in response to defective attachments of kinetochores to spindle microtubules-a mechanism called the spindle assembly checkpoint $(S A C)^{32,33}$. However, Bub1 and Bub3 also have a role in tension sensing in spindles independent of their role in the $\mathrm{SAC}^{32}$. This can be seen in the observation that bub1/3 mutants have lower Gl scores in benomyl than mad1-3 mutants (Fig. 8).

Interestingly, several other mutants did not show reduced fitness in benomyl but did display a chromosome loss phenotype (Fig. 8). These mutants were also SL or synthetic sick with bub1/3 mutants. Clb5 is one such mutant and has previously been predicted to have a role in tension sensing ${ }^{32,33}$, which the $\mathrm{Gl}$ suggests works independently of Bub1/Bub3. Interestingly, although Sic1 works to inhibit CDK-Clb ${ }^{68-70}$, including Clb5, the sic $1 \Delta$ phenotypes were similar to those of $c l b 5$ mutants. Since Sic1 is important for suppressing CDK/Clb activity and is activated by the mitotic exit network, we hypothesize that elevated $\mathrm{CDK} / \mathrm{Clb}$ may prolong anaphase resulting in spindle positioning defects, or defects in SAC silencing.

Although slow growth of swi6 $\Delta$ mutants made it difficult to assess halos, like clb5 $\Delta$ and sic $1 \Delta$ mutants, they also appeared to increase chromosome loss. However, unlike Clb5 and Sic1, Swi6 has no direct role in mitosis. Nevertheless, reduced viability in bub1/3 swi6 double mutants suggests some interaction. We propose that reduced activity of the MBF and SBF at START perturbs expression of proteins important for spindle function or chromosome cohesion, exacerbating the chromosome segregation defects of the bub1/3 mutants.

It is important to note that not all of the gene combinations that we identified as 'high-confidence' SL had remarkably negative Gl scores in our screen. There are two plausible explanations for this discrepancy. First, the use of in-plate wild-type controls prohibited the use of antibiotics in the phenotyping screen, so false negatives (growth where growth is not expected) due to contamination are more likely. Second, for gene combinations that are truly SL, any living colonies are necessarily the result of false negatives due to genetic mishaps. These gene combinations are thus more prone to result in outliers with higher than expected Gl scores and should be interpreted with caution.

\section{DISCUSSION}

The selective pressure applied by SL screens leads to genetic mishaps that rescue mutants that would otherwise be lethal ${ }^{29,35}$; conversely, the low fitness of many of the single mutant parents can add up and result in offspring with such a low fitness that they may be interpreted as SL. These false-negative and false-positive events lead to very high levels of variability (see Table S5 for an example). We accounted for this variability by probing a relatively small number of genes with an unprecedented number of biological replicates. While E-MAP screens generally incorporate four biological replicates ${ }^{24}$, and SGA screens rely on technical replicates alone ${ }^{29}$, most of the Gls tested in this study included between 8 and 16 independent biological replicates (Table S4). We also compared our results with previous publications and resolved discrepancies via TA in order to generate a list of 'high confidence' SL interactions which informed a new iteration of a previously published cell cycle model.

Variability in SL screens is a major challenge for modelers. The $\sim 100$ tetrad analyses performed in this study demonstrate an unexpectedly high level of variation even among low-throughput, manual experiments. For this reason, synthetic lethality may not be the best marker for parameterizing models. In addition, models based on synthetic lethality are inherently deterministic; yet, it is well-known that many of the processes governing progression through the cell cycle are stochastically regulated. Modeling stochasticity will require a more fine-grained dataset that provides quantitative phenotypes based on parameters such as growth rate, rather than deterministic phenotypes such as lethality or checkpoint arrest.

The scope of the 2015 Kraikivski model represents a subset of the genes involved in cell proliferation ${ }^{71-73}$. It would have been desirable to add new genes to the model so that the revised model could predict the phenotype of all the mutants included in our experiment. Some of the genes are not immediately related to the cycle regulatory network. Adding them to the 2015 model would require an extensive modeling effort. In addition, accounting for the variability of synthetic lethality data and the fitness estimates would require a very substantial modification of the 2015 modeling framework that can only predict viability and is unable to account for growth rates.

The results presented here demonstrate that quantitative cell phenotyping can be readily performed in a high-throughput workflow. By comparing colony sizes over time, we generated a quantitative picture of growth rates for over 6500 mutants. This more sensitive approach enabled us to identify interesting Gls with less extreme phenotypes than synthetic lethality (e.g., whi3 $\Delta$ ydj1 $\Delta$ ) and gene combinations that provided a rescue effect (e.g., bub3 $\Delta$ cdh $1 \Delta$ ). We also show that our workflow can be expanded to include different test conditions. By quantitatively phenotyping our mutants on six different media types, we demonstrate that our approach is sensitive enough to capture environmental variability. Data for the $\sim 40,000$ gene-by-media combinations is available in the supplement and can be used to develop more elaborate models of cell cycle regulatory control.

This potential for rapid generation of complex datasets capturing cellular response to multifactorial perturbations makes it considerably more challenging to build and update models that can explain the data and make testable, novel predictions. At one end of the spectrum are approaches that can use gene expression measurements to predict growth rates ${ }^{74,75}$. These methods can be trained rapidly but do not provide insights into underlying molecular mechanisms. In contrast, detailed, kinetic models such as the Kraikivski model encompass a wealth of molecular mechanisms. They can make predictions on the effect of multigene perturbations. However, they are challenging to parameterize, and it can be expensive to update and expand them in the face of new datasets. In order for the analysis of data and modeling to keep up with the rapid acceleration of data production it will be necessary to develop more scalable frameworks. In order for the analysis of data to keep up with the rapid acceleration of data production, it will be necessary to adopt more scalable modeling frameworks ${ }^{73,76}$.

\section{METHODS}

\section{Experimental workflow}

To generate the double mutants, we used a modified epistasis miniarray profile (E-MAP) workflow ${ }^{30}$. The E-MAP workflow is a modification of the synthetic genetic array (SGA) protocol ${ }^{30}$. In a typical SGA screen, a single query strain is crossed to all viable deletion strains (over 4000) ${ }^{29,41}$. The query strain includes a set of reporter genes that allow selection of haploid progeny of one mating type or another. E-MAP screens use the same series of selection conditions, but generally involve a few hundred deletion strains crossed to produce every possible combination of double-gene deletions $s^{30}$.

Our experimental design most closely follows the E-MAP approach but with a few significant differences. First, we focused on a set of only 36 cell cycle genes. Second, we used eight sets of parent strains in four sets of crosses, increasing the number of biological replicates to eight from four in 
a standard E-MAP or one in a standard SGA (which use technical replicates $\left.{ }^{29,30}\right)$ :
(1) MATa/gene $;:$ kanMX

(2) MATa/gene $;::$ natMX

(3) MATa/gene $::$ kanMX

(4) MATa/gene $::$ natMX
(5) MATa/gene $::$ kanMX/SGA

(6) $M A T a / g e n e_{i}:$ natMX/SGA

(7) $M A T a / g e n e_{j}:: k a n M X / S G A$

(8) $M A T a / g e n e_{i}:$ natMX/SGA gene $_{i}:$ kanMX refers to gene $e_{i}$ knocked-out with a kanamycin-resistance marker, gene $e_{i}:$ natMX refers to gene ${ }_{i}$ knocked-out with a nourseothricinresistance marker, and 'SGA' refers to the haploid-selection markers can $1 \triangle$ ::

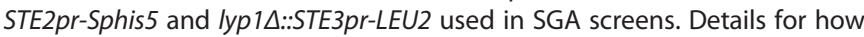
each of the parent strains were generated can be found in the online supplement. These parent strains were confirmed by PCR and used in four sets of crosses:

Cross 1: Strain $\left(1\right.$, gene $\left._{i}\right) \times \operatorname{Strain}\left(8\right.$, gene $\left._{k}\right)$

Cross 2: $\operatorname{Strain}\left(2\right.$, gene $\left._{i}\right) \times \operatorname{Strain}\left(7\right.$, gene $\left._{k}\right)$

Cross 3: $\operatorname{Strain}\left(3\right.$, gene $\left._{i}\right) \times \operatorname{Strain}\left(6\right.$, gene $\left._{k}\right)$

Cross 4: Strain $\left(4\right.$, gene $\left._{i}\right) \times \operatorname{Strain}\left(5\right.$, gene $\left._{k}\right)$

From these crosses, we selected double-mutant progeny of both mating types, further increasing the biological replicates to 16. (SGA and E-MAP screens select only MATa progeny ${ }^{29,30}$ ). All media were standard recipes for $\mathrm{SGA}^{29}$ (see online supplement). Mating type of the double mutant progeny and the single mutant parents was confirmed via Halo assays ${ }^{77}$ (See online supplement). With this protocol, we generate (in principle) 16 biological replicates of each double-mutant, gene $\Delta$ gene ${ }_{k} \Delta$. In certain cases, two parents of the same genotype were generated independently, such that the total number of biological replicates is up to 20 for some double mutant combinations.

We measured colony growth rates on three different growth media (YPD, YPG, and YPR) and in the presence of three different checkpoint activating drugs: Benomyl (Ben), Camptothecin (CPT), and Hydroxyurea (HU). Ben disrupts attachment of kinetochores to the mitotic spindle and activates the spindle assembly checkpoint (SAC; dependent on Bub1,3 and Mad1-3 $)^{50,78,79}$. CPT inhibits topoisomerase resulting in DNA entanglements and double strand breaks upon chromosome segregation ${ }^{80,81}$. HU inhibits ribonucleotide reductase ${ }^{82}$, which leads to replication fork stalling ${ }^{83}$

We first derived 384 template arrays from 96 array of the haploid progeny (which were in 96 array) using a Rotor HDA (Singer Instruments, Somerset, UK). The 96 progeny array and the 384 template array were grown on YPD $+\mathrm{G} 418(600 \mu \mathrm{g} / \mathrm{ml}) /$ nat $(150 \mu \mathrm{g} / \mathrm{ml})$. Rows $\mathrm{A}, \mathrm{B}, \mathrm{I}$ and J were left empty for in-plate wild-type controls colonies. At the same time, we set up YPD plates with the wild-type parent strains BY4741 and BY4742 arrayed at 384 density, occupying positions in rows $A, B, I$, and J. We incubated both sets of plates at $30^{\circ} \mathrm{C}$ for 2 days.

We then replica pinned the wild-type controls onto new YPD plates (using a new source plate whenever the colonies began to look depleted). After visual inspection of the plates to ensure even transfer of the wildtype controls, we replica pinned the set of double mutant colonies to the templates. Plates were imaged after $12,24,36,48$, and $60 \mathrm{~h}$ of growth at $30^{\circ} \mathrm{C}$ (Fig. S1A).

We imaged all diploid selection plates, final haploid progeny selection plates, halo assay plates, and phenotyping plates using the Phenobooth (Singer Instruments, Somerset, UK) imaging platform and software. To maintain consistency, all images were collected in the same order at the same resolution and camera settings, and were batch processed to crop the image, perform background subtraction and colony identification whenever possible. We then exported the raw colony size data for analysis.

The yeast mutants are available upon request to the corresponding author under the terms of the OpenMTA.

\section{Data analysis}

Plate-to-plate variation was accounted for by normalizing colony size using in-plate wild-type controls. Edge-effects were accounted for by adjusting the growth rates such that the mean growth rates of edge-adjacent colonies and internal colonies were comparable (Fig. S1B and Fig. S2). Jack- knife filtering was used in a small number of cases to remove colonies that behaved as outliers within quadruplicates (four technical replicates).

Growth rates, fitness scores, and Gl scores ${ }^{45-47}$ were calculated using a linear model for growth rate according to the following equations:

$$
\begin{array}{ll}
\text { Growth rate }(r): & s_{\mathrm{t}}=r \cdot t+s_{0} \\
\text { Fitness score }(W): & W=r_{\text {mutant }} / r_{\mathrm{WT}}, \\
\text { Genetic interaction score }(\varepsilon): & \varepsilon=W_{\mathrm{AB}}-W_{\mathrm{A}} W_{\mathrm{B}}
\end{array},
$$

$W_{\mathrm{AB}}=$ fitness score for the double-mutant progeny, $W_{\mathrm{A}}=$ fitness score for the MATa parent, $W_{B}=$ fitness score for the MATa mutant, $s_{\mathrm{t}}=$ colony size at time $t$, and $s_{0}=$ colony size at time 0 .

A histogram binning procedure was used to estimate the mode for $\mathrm{Gl}$ scores across biological replicates (up to 20 independent crosses). The 'consensus' Gl score reported in Figs. 7 and 8 is the midpoint of the bin containing the maximum number of values (additional details in the Supplement).

\section{Reporting summary}

Further information on experimental design is available in the Nature Research Reporting Summary linked to this article.

\section{DATA AVAILABILITY}

The complete set of data and scripts to extract genetic interactions data are available from Figshare ${ }^{84}$.

Received: 25 November 2019; Accepted: 6 April 2020;

Published online: 06 May 2020

\section{REFERENCES}

1. Morgan, D. \& Morgan, D. O. The Cell Cycle: Principles of Control. (OUP/New Science Press, 2007).

2. Csikasz-Nagy, A., Palmisano, A. \& Zamborszky, J. Molecular network dynamics of cell cycle control: transitions to start and finish. Methods Mol. Biol. 761, 277-291 (2011).

3. Alberghina, L., Hofer, T. \& Vanoni, M. Molecular networks and system-level properties. J. Biotechnol. 144, 224-233 (2009).

4. Wen, Z. et al. Identifying responsive modules by mathematical programming: an application to budding yeast cell cycle. PLOS ONE 7, e41854 (2012).

5. Ideker, T. E. Network genomics. Ernst Schering Research Foundation Workshop, 89-115 (2007)

6. Carter, G. W. et al. A systems-biology approach to modular genetic complexity. Chaos 20, 026102 (2010).

7. Ramon, C., Gollub, M. G. \& Stelling, J. Integrating -omics data into genome-scale metabolic network models: principles and challenges. Essays Biochem. 62, 563-574 (2018).

8. Trescher, S., Munchmeyer, J. \& Leser, U. Estimating genome-wide regulatory activity from multi-omics data sets using mathematical optimization. BMC Syst. Biol. 11, 41 (2017).

9. Buescher, J. M. \& Driggers, E. M. Integration of omics: more than the sum of its parts. Cancer Metab. 4, 4 (2016).

10. Bersanelli, M. et al. Methods for the integration of multi-omics data: mathematical aspects. BMC Bioinform. 17(Suppl 2), 15 (2016).

11. Yilmaz, L. S. \& Walhout, A. J. Metabolic network modeling with model organisms. Curr. Opin. Chem. Biol. 36, 32-39 (2017).

12. Hou, J., Acharya, L., Zhu, D. \& Cheng, J. An overview of bioinformatics methods for modeling biological pathways in yeast. Brief. Funct. Genomics 15, 95-108 (2016).

13. Sanchez, B. J. \& Nielsen, J. Genome scale models of yeast: towards standardized evaluation and consistent omic integration. Integr. Biol. 7, 846-858 (2015).

14. Garcia-Campos, M. A., Espinal-Enriquez, J. \& Hernandez-Lemus, E. Pathway analysis: state of the art. Front. Physiol. 6, 383 (2015).

15. Brodland, G. W. How computational models can help unlock biological systems. Semin. Cell Dev. Biol. 47-48, 62-73 (2015).

16. Soh, K. C., Miskovic, L. \& Hatzimanikatis, V. From network models to network responses: integration of thermodynamic and kinetic properties of yeast genome-scale metabolic networks. FEMS Yeast Res. 12, 129-143 (2012). 
17. Alberghina, L., Coccetti, P. \& Orlandi, I. Systems biology of the cell cycle of Saccharomyces cerevisiae: from network mining to system-level properties. Biotechnol. Adv. 27, 960-978 (2009).

18. Barberis, M., Todd, R. G. \& van der Zee, L. Advances and challenges in logical modeling of cell cycle regulation: perspective for multi-scale, integrative yeast cell models. FEMS Yeast Res 17, fow103 (2016).

19. Kraikivski, P., Chen, K. C., Laomettachit, T., Murali, T. M. \& Tyson, J. J. From START to FINISH: computational analysis of cell cycle control in budding yeast. NPJ Syst. Biol. Appl. 1, 15016 (2015).

20. Radmaneshfar, E. et al. From START to FINISH: the influence of osmotic stress on the cell cycle. PLoS ONE 8, e68067 (2013).

21. Barik, D., Ball, D. A., Peccoud, J. \& Tyson, J. J. A stochastic model of the yeast cell cycle reveals roles for feedback regulation in limiting cellular variability. PLOS Comput. Biol. 12, e1005230 (2016).

22. Chen, K. C. et al. Integrative analysis of cell cycle control in budding yeast. Mol. Biol. Cell 15, 3841-3862 (2004).

23. Ball, D. A. et al. Stochastic exit from mitosis in budding yeast: model predictions and experimental observations. Cell Cycle 10, 999-1009 (2011).

24. Ball, D. A. et al. Oscillatory dynamics of cell cycle proteins in single yeast cells analyzed by imaging cytometry. PLoS ONE 6, e26272 (2011).

25. Adames, N. R. et al. Experimental testing of a new integrated model of the budding yeast Start transition. Mol. Biol. Cell 26, 3966-3984 (2015).

26. Ball, D. A. et al. Measurement and modeling of transcriptional noise in the cell cycle regulatory network. Cell Cycle 12, 3203-3218 (2013).

27. Alberghina, L., Rossi, R. L., Wanke, V., Querin, L. \& Vanoni, M. Checking cell size in budding yeast: a systems biology approach. Ital. J. Biochem. 52, 55-57 (2003).

28. Adames, N. R., Gallegos, J. E. \& Peccoud, J. Yeast genetic interaction screens in the age of CRISPR/Cas. Curr. Genet. 65, 307-327 (2019).

29. Tong, A. H. \& Boone, C. Synthetic genetic array analysis in Saccharomyces cerevisiae. Methods Mol. Biol. 313, 171-192 (2006).

30. Schuldiner, M. et al. Exploration of the function and organization of the yeast early secretory pathway through an epistatic miniarray profile. Cell 123, 507-519 (2005).

31. Cherry, J. M. et al. SGD: saccharomyces genome database. Nucleic Acids Res. 26, 73-79 (1998).

32. Ikui, A. E. \& Cross, F. R. Specific genetic interactions between spindle assembly checkpoint proteins and B-type cyclins in Saccharomyces cerevisiae. Genetics 183, 51-61 (2009).

33. Proudfoot, K. G. et al. Checkpoint proteins Bub1 and Bub3 delay anaphase onset in response to low tension independent of microtubule-kinetochore detachment. Cell Rep. 27, 416-428 e414 (2019).

34. Hucka, M. et al. The systems biology markup language (SBML): a medium for representation and exchange of biochemical network models. Bioinformatics 19, 524-531 (2003).

35. Daniel, J. A., Yoo, J., Bettinger, B. T., Amberg, D. C. \& Burke, D. J. Eliminating gene conversion improves high-throughput genetics in Saccharomyces cerevisiae. Genetics 172, 709-711 (2006).

36. Gallegos, J. E., Adames, N. R., Hayrynen, S. \& Peccoud, J. Challenges and opportunities for strain verification by whole-genome sequencing. Sci. Rep 10, 5873 (2020).

37. Singh, I., Pass, R., Togay, S. O., Rodgers, J. W. \& Hartman, J. L. T. Stringent matingtype-regulated auxotrophy increases the accuracy of systematic genetic interaction screens with Saccharomyces cerevisiae mutant arrays. Genetics 181, 289-300 (2009).

38. Lesage, G. et al. Analysis of beta-1,3-glucan assembly in Saccharomyces cerevisiae using a synthetic interaction network and altered sensitivity to caspofungin. Genetics 167, 35-49 (2004).

39. Tong, A. H. et al. Global mapping of the yeast genetic interaction network. Science 303, 808-813 (2004).

40. Vizeacoumar, F. J. et al. Integrating high-throughput genetic interaction mapping and high-content screening to explore yeast spindle morphogenesis. J. Cell Biol. 188, 69-81 (2010)

41. Tong, A. H. et al. Systematic genetic analysis with ordered arrays of yeast deletion mutants. Science 294, 2364-2368 (2001).

42. Sarin, S. et al. Uncovering novel cell cycle players through the inactivation of securin in budding yeast. Genetics 168, 1763-1771 (2004).

43. St Onge, R. P. et al. Systematic pathway analysis using high-resolution fitness profiling of combinatorial gene deletions. Nat. Genet. 39, 199-206 (2007).

44. Bandyopadhyay, S. et al. Rewiring of genetic networks in response to DNA damage. Science 330, 1385-1389 (2010).

45. Baryshnikova, A. et al. Global linkage map connects meiotic centromere function to chromosome size in budding yeast. G3 3, 1741-1751 (2013).

46. Wagih, O. et al. SGAtools: one-stop analysis and visualization of array-based genetic interaction screens. Nucleic Acids Res. 41, W591-W596 (2013).
47. Kuzmin, E., Costanzo, M., Andrews, B. \& Boone, C. Synthetic genetic array analysis. Cold Spring Harb. Protoc. pdb.prot088807 (2016).

48. Wheals, B. B. Simple preparation of a bonded cation-exchange packing material and its application to the separation of phenothiazines by high-performance liquid chromatography. J. Chromatogr. 177, 263-270 (1979).

49. Barik, D., Ball, D. A., Peccoud, J. \& Tyson, J. J. A stochastic model of the yeast cell cycle reveals roles for feedback regulation in limiting cellular variability. PLoS Comput. Biol. 12, e1005230 (2016).

50. Li, R. \& Murray, A. W. Feedback control of mitosis in budding yeast. Cell 66, 519-531 (1991).

51. Dudley, A. M., Janse, D. M., Tanay, A., Shamir, R. \& Church, G. M. A global view of pleiotropy and phenotypically derived gene function in yeast. Mol. Syst. Biol. 1, 2005.0001 (2005).

52. Parsons, A. B. et al. Integration of chemical-genetic and genetic interaction data links bioactive compounds to cellular target pathways. Nat. Biotechnol. 22, 62-69 (2004).

53. Brown, J. A. et al. Global analysis of gene function in yeast by quantitative phenotypic profiling. Mol. Syst. Biol. 2, 2006.001 (2006).

54. Chen, S. H., Albuquerque, C. P., Liang, J., Suhandynata, R. T. \& Zhou, H. A proteome-wide analysis of kinase-substrate network in the DNA damage response. J. Biol. Chem. 285, 12803-12812 (2010).

55. Duffy, S. et al. Overexpression screens identify conserved dosage chromosome instability genes in yeast and human cancer. Proc. Natl Acad. Sci. USA 113, 9967-9976 (2016).

56. Kapitzky, L. et al. Cross-species chemogenomic profiling reveals evolutionarily conserved drug mode of action. Mol. Syst. Biol. 6, 451 (2010).

57. Shively, C. A. et al. Genetic networks inducing invasive growth in Saccharomyces cerevisiae identified through systematic genome-wide overexpression. Genetics 193, 1297-1310 (2013).

58. Wang, S. H. et al. Curcumin-mediated HDAC inhibition suppresses the DNA damage response and contributes to increased DNA damage sensitivity. PLoS ONE 10, e0134110 (2015).

59. Woolstencroft, R. N. et al. Ccr4 contributes to tolerance of replication stress through control of CRT1 mRNA poly(A) tail length. J. Cell Sci. 119, 5178-5192 (2006).

60. Wu, X. \& Jiang, Y. W. Genetic/genomic evidence for a key role of polarized endocytosis in filamentous differentiation of S. cerevisiae. Yeast 22, 1143-1153 (2005).

61. louk, T., Kerscher, O., Scott, R. J., Basrai, M. A. \& Wozniak, R. W. The yeast nuclear pore complex functionally interacts with components of the spindle assembly checkpoint. J. Cell Biol. 159, 807-819 (2002).

62. Tatchell, K., Makrantoni, V., Stark, M. J. \& Robinson, L. C. Temperature-sensitive ipl1-2/Aurora B mutation is suppressed by mutations in TOR complex 1 via the Glc7/PP1 phosphatase. Proc. Natl Acad. Sci. USA 108, 3994-3999 (2011).

63. Daniel, J. A., Keyes, B. E., Ng, Y. P., Freeman, C. O. \& Burke, D. J. Diverse functions of spindle assembly checkpoint genes in Saccharomyces cerevisiae. Genetics 172 , 53-65 (2006).

64. Doncic, A., Ben-Jacob, E., Einav, S. \& Barkai, N. Reverse engineering of the spindle assembly checkpoint. PLoS ONE 4, e6495 (2009).

65. Choi, J. E. \& Chung, W. H. Synthetic lethal interaction between oxidative stress response and DNA damage repair in the budding yeast and its application to targeted anticancer therapy. J. Microbiol. 57, 9-17 (2019).

66. Diaz-Mejia, J. J. et al. Mapping DNA damage-dependent genetic interactions in yeast via party mating and barcode fusion genetics. Mol. Syst. Biol. 14, e7985 (2018).

67. Leung, G. P., Aristizabal, M. J., Krogan, N. J. \& Kobor, M. S. Conditional genetic interactions of RTT107, SLX4, and HRQ1 reveal dynamic networks upon DNA damage in S. cerevisiae. G3 4, 1059-1069 (2014).

68. Schwob, E., Bohm, T., Mendenhall, M. D. \& Nasmyth, K. The B-type cyclin kinase inhibitor p40SIC1 controls the G1 to S transition in S. cerevisiae. Cell 79, 233-244 (1994).

69. Donovan, J. D., Toyn, J. H., Johnson, A. L. \& Johnston, L. H. P40SDB25, a putative CDK inhibitor, has a role in the M/G1 transition in Saccharomyces cerevisiae. Genes Dev. 8, 1640-1653 (1994).

70. Nugroho, T. T. \& Mendenhall, M. D. An inhibitor of yeast cyclin-dependent protein kinase plays an important role in ensuring the genomic integrity of daughter cells. Mol. Cell. Biol. 14, 3320 (1994).

71. Kaizu, K. et al. A comprehensive molecular interaction map of the budding yeast cell cycle. Mol. Syst. Biol. 6, 415 (2010).

72. Alberghina, L. et al. Cell growth and cell cycle in Saccharomyces cerevisiae: basic regulatory design and protein-protein interaction network. Biotechnol. Adv. 30, 52-72 (2012).

73. Munzner, U., Klipp, E. \& Krantz, M. A comprehensive, mechanistically detailed, and executable model of the cell division cycle in Saccharomyces cerevisiae. Nat. Commun. 10, 1308 (2019) 
74. Airoldi, E. M. et al. Predicting cellular growth from gene expression signatures. PLoS Comput. Biol. 5, e1000257 (2009).

75. Wytock, T. P. \& Motter, A. E. Predicting growth rate from gene expression. Proc. Natl Acad. Sci. USA 116, 367-372 (2019).

76. Camacho, D. M., Collins, K. M., Powers, R. K., Costello, J. C. \& Collins, J. J. Nextgeneration machine learning for biological networks. Cell 173, 1581-1592 (2018)

77. Sprague, G. F. Jr. Assay of yeast mating reaction. Methods Enzymol. 194, 77-93 (1991).

78. Hoyt, M. A., Totis, L. \& Roberts, B. T. S. cerevisiae genes required for cell cycle arrest in response to loss of microtubule function. Cell 66, 507-517 (1991).

79. Silva, P. et al. Monitoring the fidelity of mitotic chromosome segregation by the spindle assembly checkpoint. Cell Prolif. 44, 391-400 (2011).

80. Eng, W. K., Faucette, L., Johnson, R. K. \& Sternglanz, R. Evidence that DNA topoisomerase $\mathrm{I}$ is necessary for the cytotoxic effects of camptothecin. Mol. Pharm. 34, 755-760 (1988).

81. Kjeldsen, E., Svejstrup, J. Q., Gromova, I. I., Alsner, J. \& Westergaard, O. Camptothecin inhibits both the cleavage and religation reactions of eukaryotic DNA topoisomerase I. J. Mol. Biol. 228, 1025-1030 (1992).

82. Turner, M. K., Abrams, R. \& Lieberman, I. Meso-alpha, beta-diphenylsuccinate and hydroxyurea as inhibitors of deoxycytidylate synthesis in extracts of Ehrlich ascites and L cells. J. Biol. Chem. 241, 5777-5780 (1966).

83. Cortez, D. Preventing replication fork collapse to maintain genome integrity. DNA Repair 32, 149-157 (2015).

84. Gallegos, J. E., Adames, N. R., Rogers, M. F., Ibele, A. \& Peccoud, J. Growth rates of 7,350 yeast cell cycle mutants measured in six different growth media. Figshare https://doi.org/10.6084/m9.figshare.11288633.v3 (2019).

\section{ACKNOWLEDGEMENTS}

This work was supported by NIH grant GM078989 and NSF Awards DBI-1759900, DBI1759858, and CMMI-1832320.

\section{AUTHOR CONTRIBUTIONS}

J.E.G. performed the experiments, analyzed the data, wrote the paper. N.R.A. designed the experiments, performed the experiments, analyzed the data, and wrote the paper. M.F.R. analyzed the data. P.K. analyzed the data. A.I., K.N.L., and E.K. performed the experiments. T.M.M. secured funding and wrote the paper. J.J.T. secured the funding and wrote the paper. J.P. secured the funding, designed the experiment, and wrote the paper.

\section{COMPETING INTERESTS}

J.P. holds an equity stake in GenoFAB, Inc. and M.R. is an employee of GenoFAB, Inc. GenoFAB may benefit or may be perceived to benefit from the publication of this article.

\section{ADDITIONAL INFORMATION}

Supplementary information is available for this paper at https://doi.org/10.1038/ s41540-020-0134-z.

Correspondence and requests for materials should be addressed to J.P.

Reprints and permission information is available at http://www.nature.com/ reprints

Publisher's note Springer Nature remains neutral with regard to jurisdictional claims in published maps and institutional affiliations.

Open Access This article is licensed under a Creative Commons Attribution 4.0 International License, which permits use, sharing, adaptation, distribution and reproduction in any medium or format, as long as you give appropriate credit to the original author(s) and the source, provide a link to the Creative Commons license, and indicate if changes were made. The images or other third party material in this article are included in the article's Creative Commons license, unless indicated otherwise in a credit line to the material. If material is not included in the article's Creative Commons license and your intended use is not permitted by statutory regulation or exceeds the permitted use, you will need to obtain permission directly from the copyright holder. To view a copy of this license, visit http://creativecommons. org/licenses/by/4.0/.

(c) The Author(s) 2020 